\title{
USP18 recruits USP20 to promote innate antiviral response through deubiquitinating STING/MITA
}

\author{
Man Zhang ${ }^{1,{ }^{*}}$, Meng-Xin Zhang ${ }^{1, *}$, Qiang Zhang ${ }^{1}$, Gao-Feng Zhu ${ }^{1}$, Lei Yuan ${ }^{1}$, Dong-Er Zhang ${ }^{4}$, Qiyun Zhu ${ }^{3}$, \\ Jing Yao ${ }^{1}$, Hong-Bing Shu ${ }^{2}$, Bo Zhong ${ }^{1,2}$ \\ ${ }^{I}$ State Key Laboratory of Virology, Hubei Key Laboratory of Cell Homeostasis, College of Life Sciences, Wuhan University, Wuhan \\ 430072, China; ${ }^{2}$ Medical Research Institute, School of Medicine, Wuhan University, Wuhan 430071, China; ${ }^{3}$ State Key Laboratory \\ of Veterinary Etiological Biology, Lanzhou Veterinary Research Institute, Chinese Academy of Agricultural Sciences, Lanzhou \\ 730046, China; ${ }^{4}$ Department of Pathology and Division of Biological Sciences, Moores UCSD Cancer Center, University of Cali- \\ fornia San Diego, La Jolla, California 92093, USA
}

STING (also known as MITA) mediates the innate antiviral signaling and ubiquitination of STING is key to its function. However, the deubiquitination process of STING is unclear. Here we report that USP18 recruits USP20 to deconjugate K48-linked ubiquitination chains from STING and promotes the stability of STING and the expression of type I IFNs and proinflammatory cytokines after DNA virus infection. USP18 deficiency or knockdown of USP20 resulted in enhanced K48-linked ubiquitination and accelerated degradation of STING, and impaired activation of IRF3 and NF-KB as well as induction of downstream genes after infection with DNA virus HSV-1 or transfection of various DNA ligands. In addition, $U s p 18^{-/-}$mice were more susceptible to $\mathrm{HSV}-1$ infection compared with the wildtype littermates. USP18 did not deubiquitinate STING in vitro but facilitated USP20 to catalyze deubiquitination of STING in a manner independent of the enzymatic activity of USP18. In addition, reconstitution of STING into Usp $18^{-/-}$MEFs restored HSV-1-induced expression of downstream genes and cellular antiviral responses. Our findings thus uncover previously uncharacterized roles of USP18 and USP20 in mediating virus-triggered signaling and contribute to the understanding of the complicated regulatory system of the innate antiviral responses.

Keywords: USP18; USP20; STING/MITA; deubiquitination; innate antiviral response Cell Research (2016) 26:1302-1319. doi:10.1038/cr.2016.125; published online 1 November 2016

\section{Introduction}

The innate immune system deploys germline-encoded pattern-recognition receptors (PRRs) to detect structurally conserved molecules known as pathogen-associated molecular patterns (PAMPs) from invading microbes [1]. After binding to PAMPs, PRRs recruit adaptor proteins and activate a series of signaling cascades that lead to the induction of downstream genes. Among the PAMPs characterized, nucleic acids including DNA and RNA are

*These two authors contributed equally to this work.

Correspondence: Bo Zhong

Tel: 86-27-68752202; Fax: 86-27-68752246

E-mail: zhongbo@whu.edu.cn

Received 17 June 2016; revised 11 July 2016; accepted 14 September 2016; published online 1 November 2016 recognized as major viral PAMPs generated during viral infection and replication [2]. Detection of cytosolic RNA depends on RIG-I and MDA5 (known as RIG-I-like receptors (RLRs)) which recruit the mitochondrial adaptor protein MAVS (also called VISA, IPS-1 or Cardif), resulting in formation of the prion-like structure of MAVS for signaling transduction [3-7]. In contrast, multiple sensors including RNA polymerase III, DAI, IFI16, DDX41 and Lsm14A have been reported to detect cytosolic DNA in cell type- and/or ligand-dependent manners [8-13]. Recently, the nucleotidyl transferase cyclic AMP-GMP synthase (cGAS) has been identified as a new sensor for cytosolic DNA [14], and gene deletion studies suggest that cGAS is critical for host defense against DNA viruses in mice as well as in various types of cells $[15,16]$, indicating that cGAS is a universal sensor for DNA viruses. Upon binding to DNA, cGAS catalyzes the synthesis of cyclic dinucleotide (cGAMP) of 2'-5'-linkage phos- 
phodiester which serves as a second messenger and binds to the endoplasmic reticulum (ER) and mitochondrial adaptor protein STING (also called MITA, MPYS and ERIS) [17-22]. Binding to cyclic di-nucleotides results in dimerization or oligomerization of STING [23-25]. The oligomers of MAVS and STING further recruit adaptor proteins such as TRAF3 and TRAF6, and kinases TBK1/ IKK $\varepsilon$ and $\mathrm{IKK} \alpha / \beta / \gamma$ complex to activate transcription factors IRF3/7 and NF- $\mathrm{KB}$ to induce expression of a large array of downstream genes including type I interferons (IFNS), CCL5, TNF and IL-6.

In addition to oligomerization, viral infection results in ubiquitination of MAVS and STING, which is essential for cellular antiviral responses. For example, MAVS is ubiquitinated by the PCBP2-AIP4 complex or by RNF5 and degraded through the ubiquitin-proteasome pathway [26, 27]. RNF5 catalyzes K48-linked ubiquitination of STING, causing the proteasome-dependent degradation of STING [28] and thus turning down the excessive immune responses, whereas RNF26 catalyzes K11-linked ubiquitination of STING on the same site, antagonizing RNF5-mediated degradation of STING [29]. On the other hand, TRIM32 and TRIM56 target STING for K63linked ubiquitination, which is critical for expression of downstream genes after viral infection [30, 31]. Recently, it has been demonstrated that the INSIG1-AMFR E3 ligase complex mediates K27-linked polyubiquitin of STING, and thereby promotes recruitment of TBK 1 to activate IRF3 [32]. However, how deubiquitination of STING is mediated is unclear.

Deubiquitination is the reverse process of ubiquitination and mediated by a group of proteins called deubiquitinating enzymes (DUBs) which consist of more than 90 family members [33]. Ubiquitin-specific protease (USP) 18 and USP20 belong to the USP subfamily of DUBs and mediate deubiquitination of targets. USP20 targets various proteins including HIF $1 \alpha$, beta adrenergic receptor, TRAF6, and Claspin for deubiquitination and thereby regulates TLR4 signaling and DNA damage repair [34-38]. USP18 was initially identified as a DUB and later characterized as a deISGylation enzyme $[39,40]$, as deficiency of USP18 or knockin of an enzyme-inactive mutant USP18 (C61A) in mice results in hyper-ISGylation [41, 42]. USP18 directly binds to interferon $\alpha$ receptor (IFNAR) subunit IFNAR2 and negatively regulates type I IFN-triggered signaling by blocking JAK1 binding to IFNAR2 [43]. Consequently, USP18 deficiency enhances viral resistance after type I IFN stimulation. However, deletion of neither ISG15 nor Ube1L in Usp $18^{-/-}$background impairs viral resistance compared with Usp $18^{-/}$cells or mice [44, 45]. Recently, it has been reported that Usp $18^{\mathrm{C} 61 \mathrm{~A}}$ cells are more resistant to influenza B virus infection compared with wild-type cells after type I IFN treatment, and deletion of ISG15 in Usp $18^{\mathrm{C} 61 \mathrm{~A}}$ background fully restores viral replication [42], indicating that USP18 regulates type I IFN-mediated antiviral responses through both deISGylation-dependent and -independent mechanisms. In addition to deISGylation, USP18 deconjugates K63-linked polyubiquitin chains from the TAK1/TABs complex downstream of TCR or TNF signaling and limits hyper-activation of NF- $\mathrm{KB}$ and NFAT $[46,47]$. Thus, USP18 exerts deISGylation, deubiquitination and non-enzymatic activities in distinct signaling pathways. Whether and how USP18 is involved in viral infection-triggered signaling has not been explored.

In this study, we identified USP18 as a STING-interacting protein. USP18 deficiency impaired HSV-1- or cytoplasmic DNA-triggered activation of IRF3 and NF$\kappa \mathrm{B}$ and subsequent induction of type I IFNs and pro-inflammatory cytokines in the presence or absence of IFNAR1. In addition, Usp $18^{-/-}$mice were more susceptible to HSV-1 infection. Interestingly, the enzymatic activity of USP18 is dispensable for virus-triggered induction of type I IFNs and pro-inflammatory cytokines. USP18 interacts with and recruits USP20 to STING and USP20 mediates deconjugation of K48-linked polyubiquitin chains from STING. Deficiency of USP18 or knockdown of USP20 resulted in hyperubiquitination and accelerated degradation of STING as well as increased replication of HSV-1. Our findings thus uncover a previously uncharacterized role of USP 18 and USP20 in regulating virus-triggered STING-mediated type I IFN induction and contribute to the complicated regulatory mechanisms of innate cellular antiviral responses.

\section{Results}

\section{Identification of USP18 as a STING-interacting protein}

Because STING undergoes various ubiquitin modifications which are essential for its function, we hypothesized that DUB-mediated deubiquitination of STING is equally important as ubiquitination and should be tightly regulated. To test this hypothesis, we performed an unbiased screening of STING-interacting DUBs by cotransfection of individual FLAG-tagged DUBs and HA-STING into HEK293 cells followed by coimmunoprecipitation and immunoblot assays. We found that $\sim 10$ DUBs examined interacted with STING, including USP18, USP20 and USP49 (Figure 1A and Supplementary information, Figure S1A). It has been reported that USP18 negatively regulates type I IFN-triggered signaling [43] and STING is critical for viral infection-triggered type I IFN induction $[19,20]$. We thus further examined the endogenous association between USP18 and STING and 
A

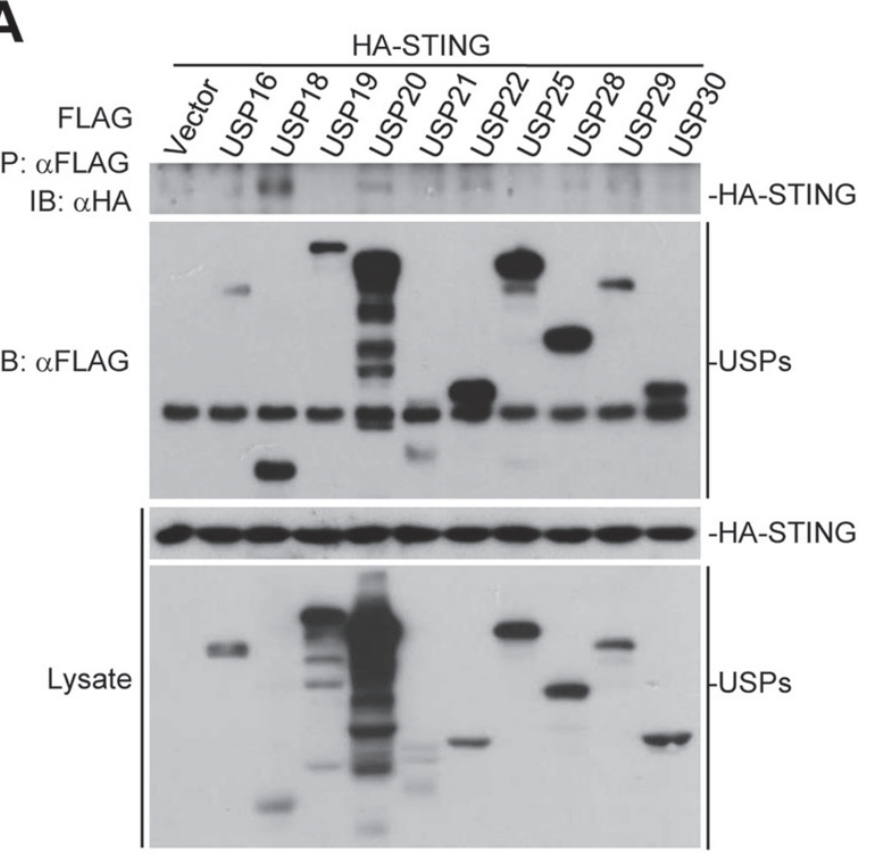

C

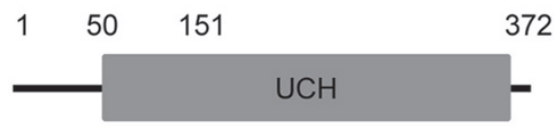

HA-STING

FLAG-USP18: 1-150 151-372 51-372 1-372

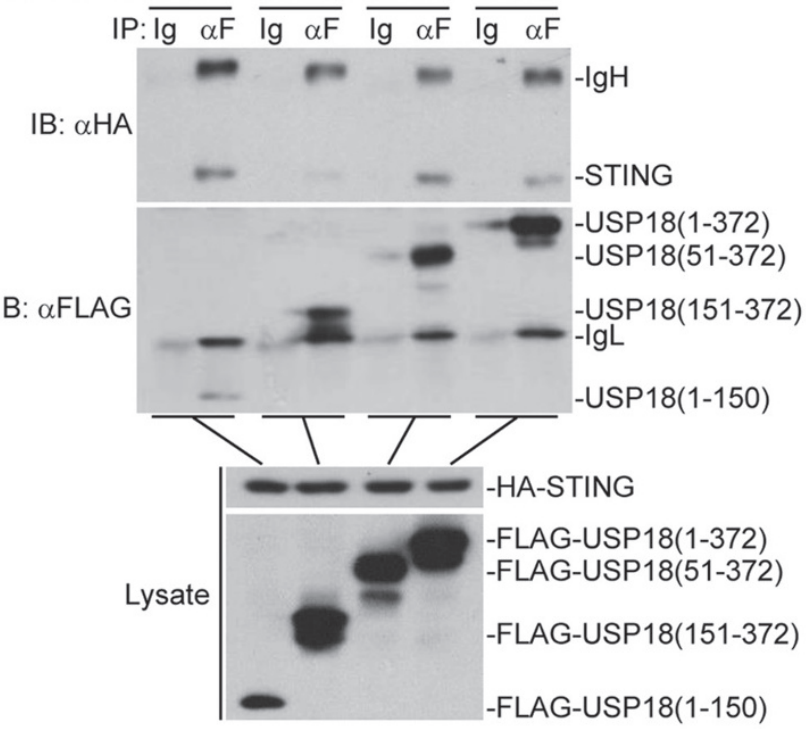

B
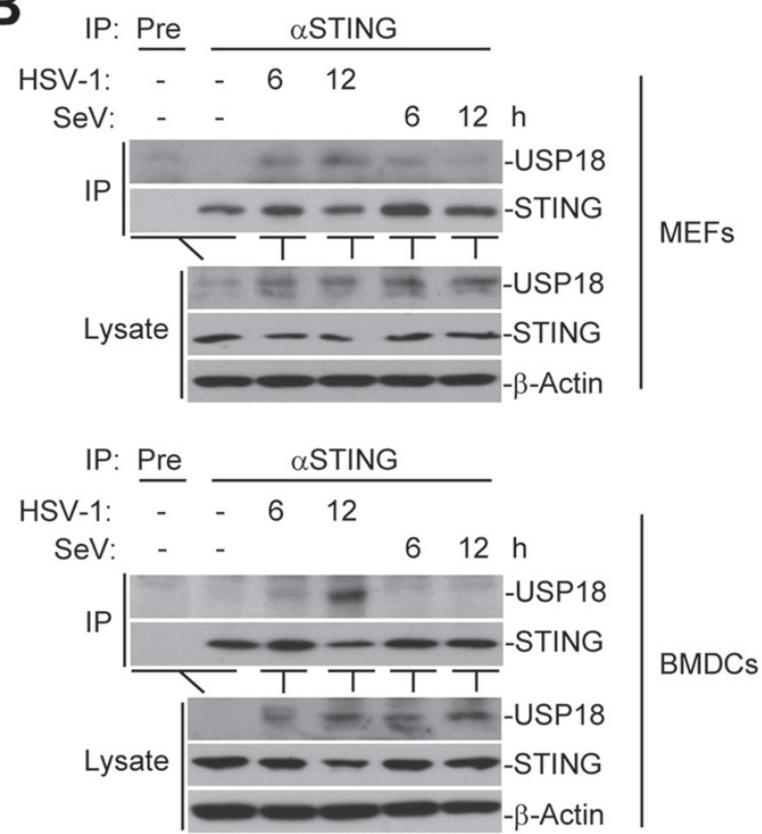

D

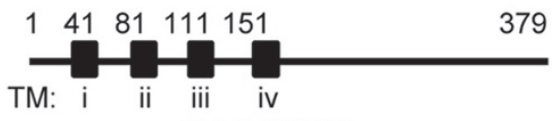

FLAG-USP18

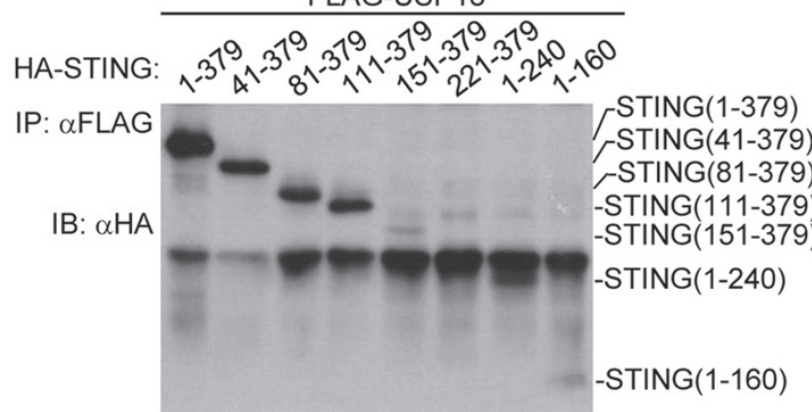

IB: $\alpha$ FLAG $=0-\infty-\infty$-FLAG-USP18

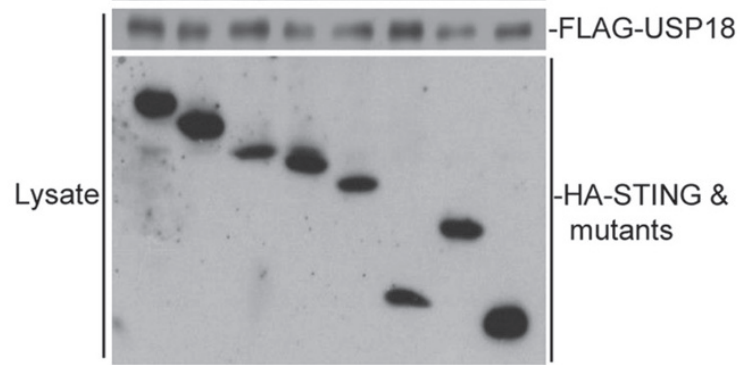

Figure 1 Identification of USP18 as a STING-interacting protein. (A) Immunoblot of HEK293 cells that were transfected to express HA-STING and FLAG-tagged DUBs, lysed and immunoprecipitated (IP) with anti-FLAG. Cell lysate was analyzed by immunoblot with anti-FLAG or anti-HA. (B) Immunoblot of MEFs (upper panels) and BMDCs (lower panels) that were left uninfected or infected with HSV-1 or SeV for 6-12 h, lysed and immunoprecipitated with a control immunoglobulin G (IgG), or anti-STING followed by immunoblot analysis with antibodies to the indicated proteins. (C, D) Immunoblot of HEK293 cells that were transfected to express HA-STING and FLAG-tagged WT or mutant USP18 (C) or FLAG-USP18 and HA-tagged WT or mutant STING (D), lysed and immunoprecipitated with anti-FLAG. Cell lysate was analyzed by immunoblot with anti-FLAG or anti-HA. Data are representative of three (A, B) or two (C, D) independent experiments. 
found that USP18 interacted with STING in mouse embryonic fibroblasts (MEFs), mouse bone marrow-derived dendritic cells (BMDCs) and THP-1 cells after DNA virus HSV-1 infection (Figure 1B and Supplementary information, Figure S1B). In contrast, RNA virus $\mathrm{SeV}$ infection induced weak and transient USP18-STING association in MEFs, and IFN- $\beta$ treatment which results in slight increase in USP18 expression level did not induce USP18-STING association in THP-1 cells, indicating that USP18 interacts with STING in a viral infection-specific manner (Figure 1B and Supplementary information, Figure S1B). Results from domain mapping analysis suggest that the intermediate domain of USP18 (aa 51-150) is essential for USP18-STING interaction, whereas the $\mathrm{N}$-terminal transmembrane domain of STING (aa 1-160) is responsible for its interaction with USP18 (Figure 1C-1D). These data suggest that USP18 interacts with STING after viral infection.

\section{USP18 deficiency impairs virus-triggered signaling}

Because STING is essential for virus infection-induced signaling, we examined whether USP18 plays a role in virus-triggered expression of downstream genes by quantitative real-time PCR (qRT-PCR) analysis. The results showed that the induction of Ifnb, Ifna 4 and Tnf was significantly dampened in Usp $18^{-/-}$BMDCs, bone marrow-derived macrophages (BMDMs) and mouse lung fibroblasts (MLFs) compared with the wild-type counterparts after infection with DNA viruses HSV-1 or HCMV, or transfection of various DNA ligands (Figure $2 \mathrm{~A}-2 \mathrm{C})$. Such inhibition was also observed in Usp $18^{-/-}$ cells infected with RNA viruses $\mathrm{SeV}$ or EMCV, although to a less extent compared with that upon DNA virus infection (Figure 2A-2C). NF- $\mathrm{KB}$ and IRF3 are two transcription factors that are essential for virus-induced expression of downstream genes. Results from immunoblot analysis showed that HSV-1-induced phosphorylation of IRF3 and I $\mathrm{B} \alpha \alpha$ was substantially impaired. EMCV-induced phosphorylation of IRF3 was slightly inhibited in Usp $18^{-/-}$BMDCs or MLFs compared with the wild-type counterparts (Figure 2D-2E). Furthermore, USP18 deficiency potentiated the replication of HSV-1 in BMDCs and MEFs (Figure 2F). These data suggest that USP18 is required for optimal induction of type I IFNs and pro-inflammatory cytokines after viral infection and plays a major role in DNA virus-triggered signaling.

\section{USP18-deficient mice are more susceptible to HSV-1 in-} fection

To characterize the role of USP18 in DNA virus infection in vivo, we infected wild-type and Usp $18^{-/-}$mice with HSV-1 by tail vein injection and monitored their survival. Consistent with the results from gene induction analysis, Usp $18^{-/-}$mice exhibited increased susceptibility to lethal HSV-1 infection compare with the wildtype littermates (Figure 3A). In addition, the induction of IFN- $\alpha$, IL- 6 and Cxcl1 was significantly decreased in the sera of Usp $18^{-/-}$mice compared with $U s p 18^{+/+}$mice at $12 \mathrm{~h}$ after HSV-1 infection (Figure 3B). The expression of type I IFNs and IL-6 was severely impaired and the replication of HSV-1 (as monitored by the expression of HSV-1 UL30 gene and the virus titers) was exacerbated in lungs, brains or kidneys from Usp $18^{-/-}$mice compared with $U s p 18^{+/+}$mice at $24 \mathrm{~h}$ or 4 days after HSV-1 infection (Figure 3C-3E). Hematoxylin-eosin staining analysis of lung tissues showed that there were less infiltrated cells in the lungs of Usp1 $18^{-/-}$mice than in the lungs of $U_{s p} 18^{+/+}$mice after HSV-1 infection (Figure 3F). These results together suggest that USP18 positively regulates virus-induced expression of downstream genes and is essential for host defense against DNA viruses in vivo.

\section{USP18 functions independently of type I IFN-triggered signaling}

Because USP18 is known to inhibit type I IFN-triggered signaling by interfering with JAK1 and IFNAR2 binding, we investigated whether USP18 regulates virus-triggered induction of downstream genes through the IFN-IFNAR pathway. To test this, we obtained $U_{s}$ p $18^{+/+}$Ifnar $^{-/-}$and Usp $18^{-/-}$Ifnar $^{-/-}$mice by crossing the Usp $18^{+/-}$Ifnar $^{-/-}$mice. The Usp $18^{-/-}$Ifnar $^{-/-}$mice developed normally and did not show any defects in immune cell homeostasis in thymus, spleen or peripheral lymph nodes (Supplementary information, Figure S2A-S2D). In addition, Usp $18^{-/-}$Ifnar $1^{-/}$bone marrow cells differentiated into DCs or macrophages normally as did Usp $18^{+/ t}$ Ifnar $1^{-/-}$bone marrow cells, indicating that USP18 deficiency in Ifnarl ${ }^{-/}$background did not affect development and homeostasis of immune cells (Supplementary information, Figure S2E-S2F). Interestingly, the induction of type I IFNs and proinflammatory cytokines was substantially impaired in Usp $18^{-/-}$Ifnar $1^{-/}$BMDCs or MEFs compared with Usp $18^{+/+}$Ifnar $1^{-/-}$counterparts after infection with HSV-1, HCMV, SeV, or EMCV or transfection of DNA ligands (Supplementary information, Figure S3A). In addition, HSV-1-induced phosphorylation of I $\mathrm{B} \alpha$ and IRF3 was impaired in Usp $18^{-/-}$Ifnar $^{-/}$ BMDCs or MEFs compared with Usp $18^{+/+}$Ifnar $^{-/-}$counterparts (Supplementary information, Figure S3B). To examine the role of USP18 in cellular antiviral responses, we infected Usp $18^{-/-}$Ifnar $1^{-/-}$or Usp $18^{+/+}$Ifnar $^{-/-}$cells with HSV-1 or GFP-tagged HSV-1 and found that the replication of HSV-1 was potentiated in Usp $18^{-/-}$Ifnar $1^{-1-}$ cells compared with the Usp $18^{+/+}$Ifnar $^{-/-}$counterparts as monitored by the expression of GFP or HSV-1 UL30 
A

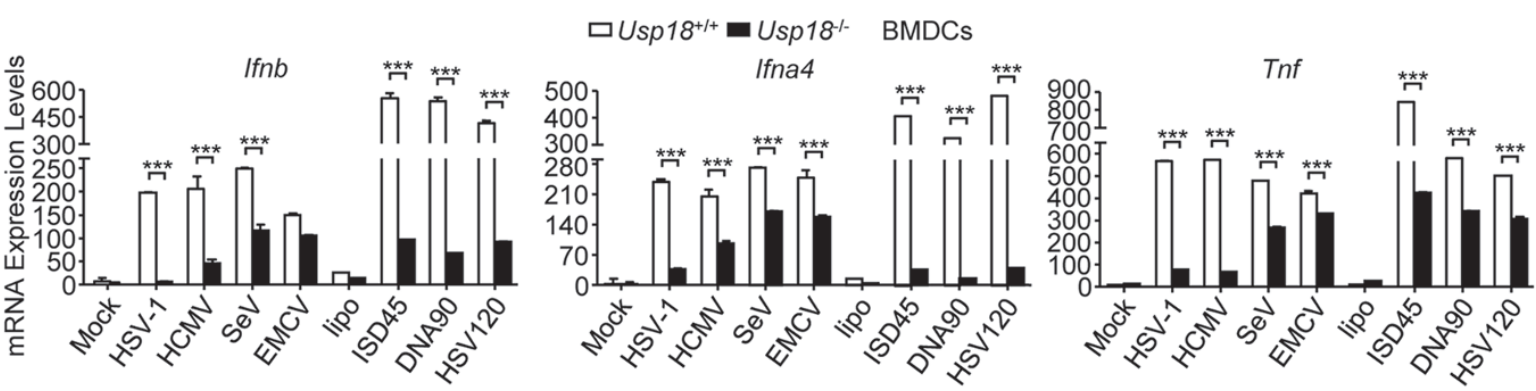

B

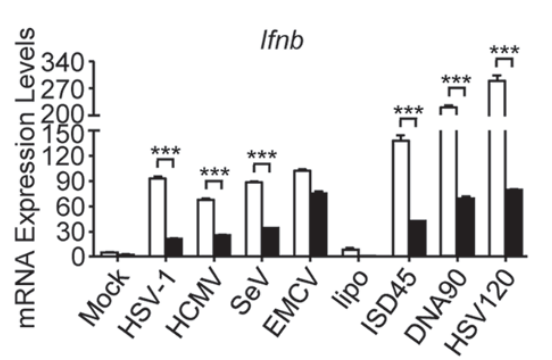

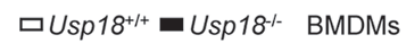
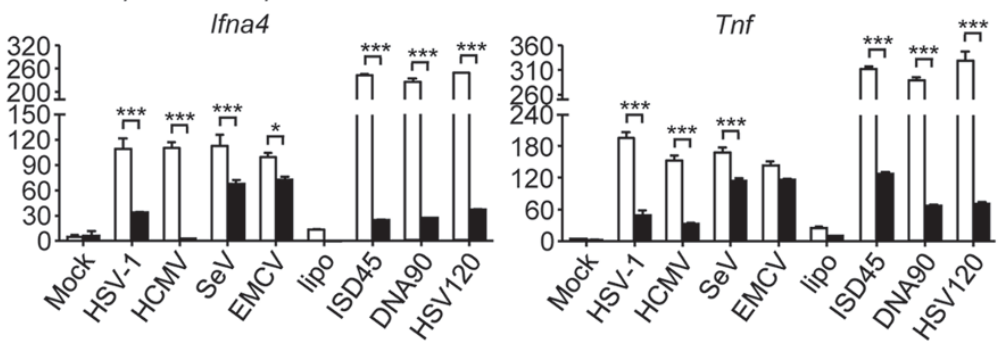

C

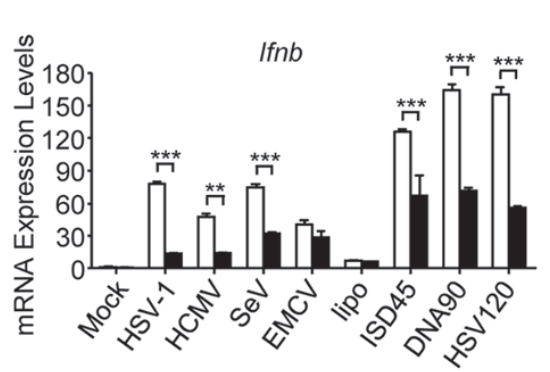

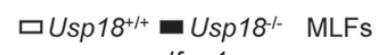

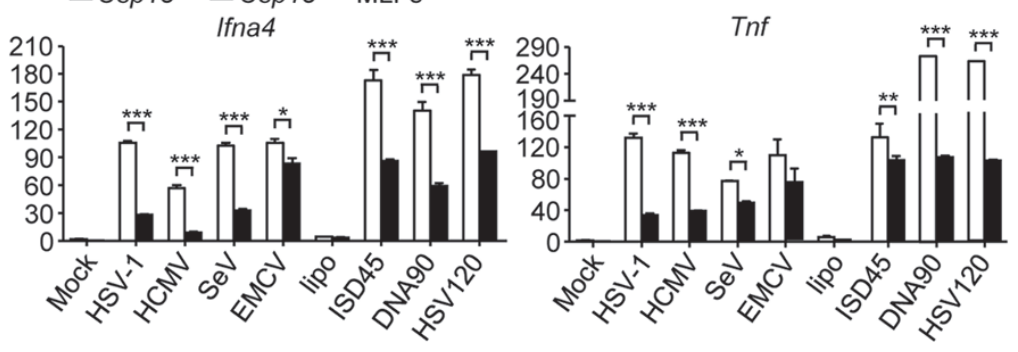

D

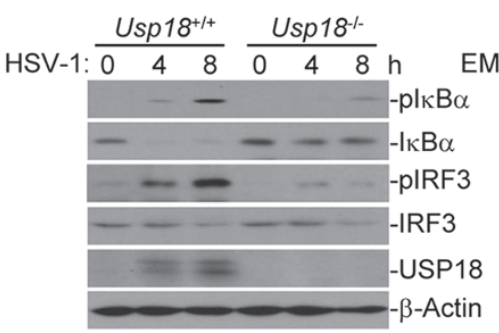

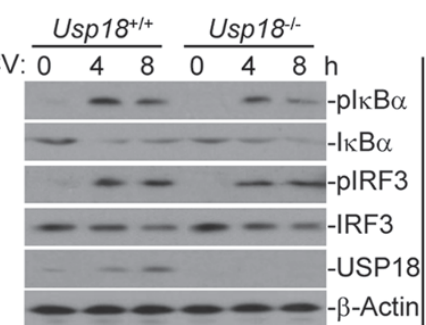

E
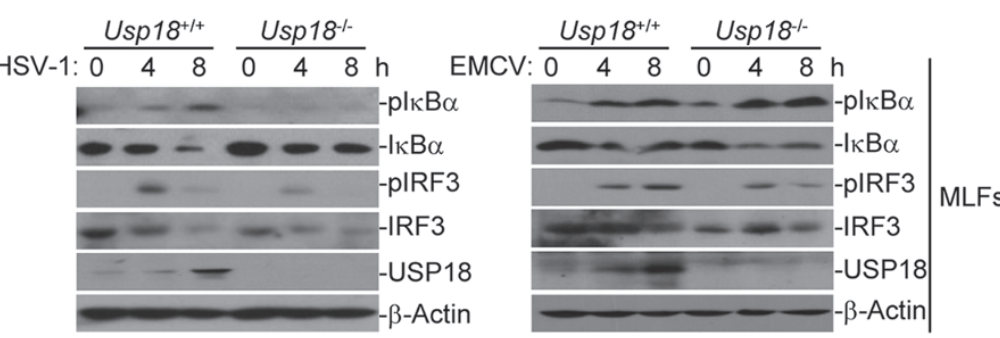

F
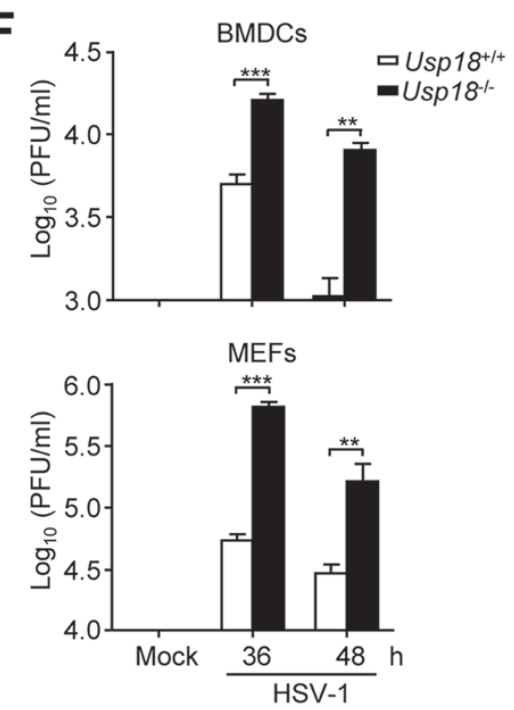

Figure 2 USP18 deficiency impairs virus-triggered signaling. (A-C) qRT-PCR analysis of Ifnb, Ifna4, Tnf mRNA in Usp18 ${ }^{+/+}$ and Usp $18^{-/-}$BMDCs $\left(5 \times 10^{5} ; \mathbf{A}\right)$, BMDMs $\left(5 \times 10^{5} ; \mathbf{B}\right)$ or MLFs $\left(1 \times 10^{5} ; \mathbf{C}\right)$ left uninfected (Mock) or untransfected (Lipo), or infected with HSV-1, HCMV, SeV, EMCV for $6 \mathrm{~h}$, or transfected with ISD45, DNA90 or HSV120 (3 $\mu \mathrm{g})$. (D, E) Immunoblot analysis of total and phosphorylated (p-) IKB $\alpha$, IRF3, total USP18, $\beta$-Actin in Usp $18^{+/+}$and Usp $18^{-/-}$BMDCs $\left(5 \times 10^{6}\right.$; D) or MLFs $\left(2 \times 10^{6}\right.$; E) infected with HSV-1 (left) or EMCV (right) for 0-8 h. (F) Plaque analysis of supernatants of Usp18 ${ }^{++}$and Usp $18^{-1-}$ BMDCs $\left(5 \times 10^{5}\right)$ or MEFs $\left(2 \times 10^{5}\right)$ that were infected with HSV-1 $(\mathrm{MOI}=0.05)$ for $1 \mathrm{~h}$ followed by PBS wash twice and cultured for 36-48 h. ${ }^{*} P<0.05$, ${ }^{* *} P<0.01$ and ${ }^{* *} P<0.001$ (analysis of two-way ANOVA followed by Bonferroni posttest). Data are representative of four (A-C) or three (D-F) independent experiments (mean \pm SD in $\mathbf{A}-\mathbf{C}$ and $\mathbf{F}$ ). 
A

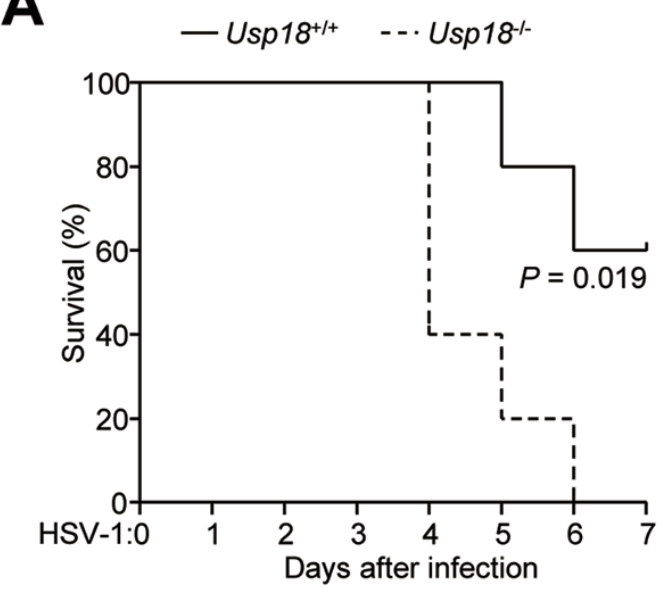

C

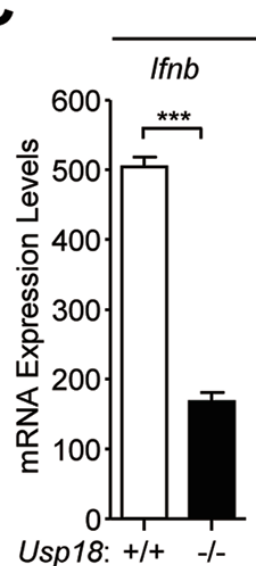

B

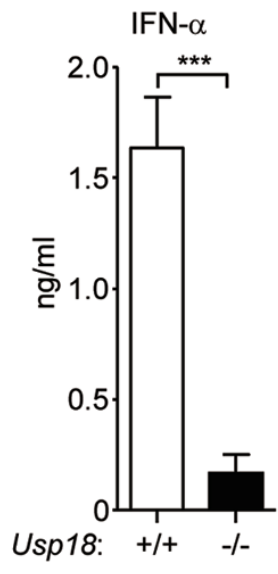

IL-6

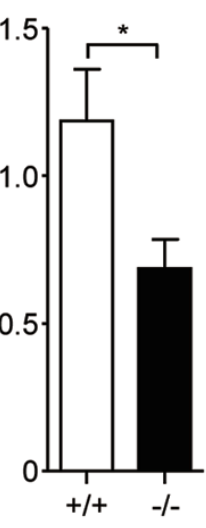

Cxcl1

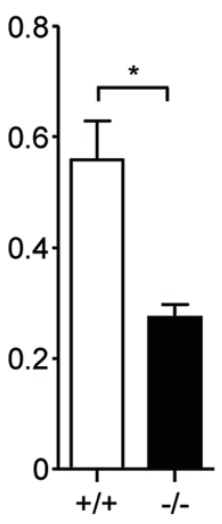

D

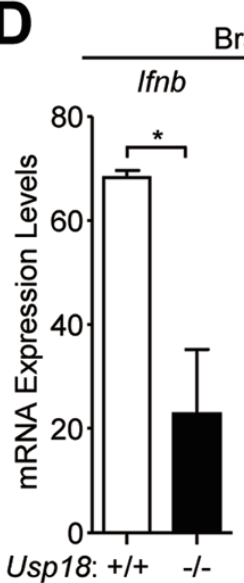

Brain

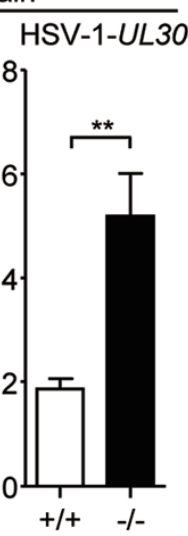

$E$

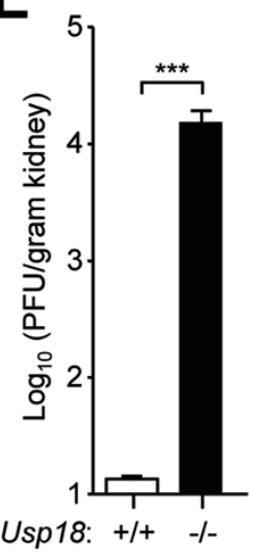

$\mathbf{F}$
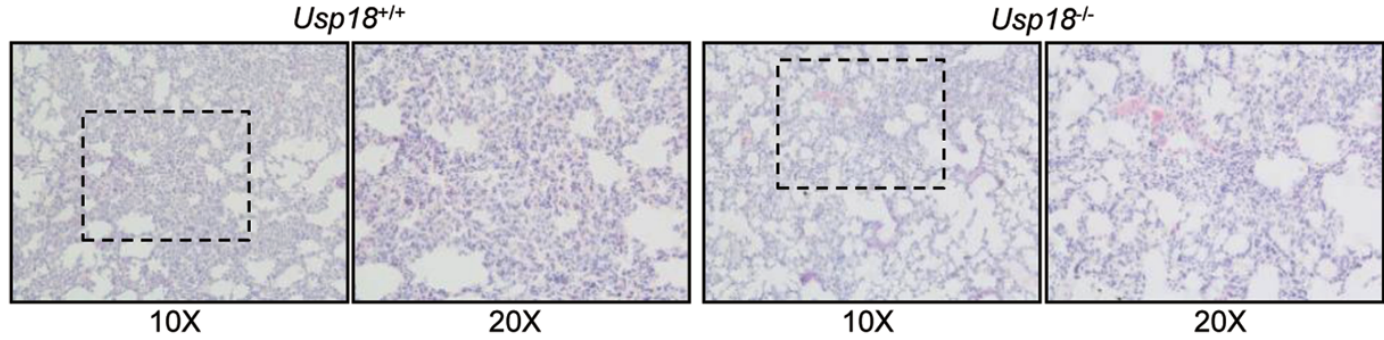

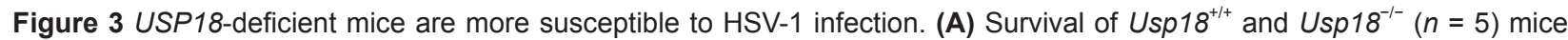
after intravenous injection of HSV-1 ( $8 \times 10^{5}$ PFU per mouse) and monitored for 7 days. (B-D) ELISA analysis of sera (B, at $12 \mathrm{~h}, n=4)$ and qRT-PCR analysis of lungs (C, at $24 \mathrm{~h}, n=3)$ or brain (D, at 4 days, $n=3)$ from Usp18 ${ }^{+/+}$and $U s p 18^{-/-}$mice injected intravenously with HSV-1 $\left(8 \times 10^{5} \mathrm{PFU}\right.$ per mouse). (E) Plaque analysis of homogenizes of kidneys from Usp $18^{+/+}$ and Usp $18^{-/-}$mice $(n=4)$ injected intravenously with HSV-1 $\left(4 \times 10^{6} \mathrm{PFU}\right.$ per mouse) for 4 days. (F) Hematoxylin-eosin staining of lungs from Usp18 $8^{+/+}$and Usp18 $8^{-/-}$mice $(n=3)$ infected with HSV-1 $\left(8 \times 10^{5}\right.$ PFU per mouse) for $24 \mathrm{~h} .{ }^{*} P<0.05,{ }^{* *} P<0.01$ and ${ }^{* *} P<0.001$ (student's $t$-test). Data are representative of three independent experiments (mean \pm SD in B-E).

gene (Supplementary information, Figure S3C-3D).

We next injected HSV-1 into Usp $18^{-/-}$Ifnar $^{-/-}$and $U s-$ $p 18^{+/ t}$ Ifnar $^{-/-}$mice through tail vein and examined expression of type I IFNs and proinflammatory cytokines in vivo. The results showed that the IFN- $\alpha$ and IL- 6 protein levels and the induction of Ifnb, Il6, and Cxcll were significantly impaired in the sera or the lungs from Usp $18^{-1}$ Ifnar $I^{-/-}$mice compared with those in Usp $18^{+/+}$Ifnar $^{-1}$ mice at 12 or $24 \mathrm{~h}$ after HSV-1 infection (Supplementary information, Figure S4A-S4B). Hematoxylin-eosin stain- 
ing analysis of lung tissues showed that less infiltrated cells existed in the lungs of Usp $18^{-/}$Ifnar $I^{-/}$mice than in the lungs of Usp $18^{+/ \mid}$Ifnar $1^{-/}$mice after HSV-1 infection (Supplementary information, Figure S4C). These data collectively suggest that USP18 mediates virus-triggered induction of downstream genes and restricts viral replication independently of the IFN-IFNAR signaling.

\section{USP18 mediates antiviral signaling independently of its DUB activity}

Considering that USP18 functions through both enzymatic and non-enzymatic activities, we next determined whether the enzymatic activity of USP18 is required for mediating virus-triggered signaling. Interestingly, reconstitution of either USP18 or USP18 (C61A) into Usp $18^{-/-}$ MEFs restored HSV-1-, HCMV- or cytosolic DNA-induced expression of Ifnb, Ifna 4, Tnf, Il6 or Cxcll (Figure $4 \mathrm{~A})$. In addition, HSV-1-induced phosphorylation of I $\mathrm{B} \alpha$ and IRF3 was restored in Usp $18^{-/-}$MEFs reconstituted with USP18 or USP18 (C61A) but not in those reconstituted with the empty vector (Figure 4B). Consistent with these observations, the enhanced replication of HSV-1 or GFP-tagged HSV-1 in Usp $18^{-/-}$MEFs was reversed by reconstitution of either USP 18 or USP 18 (C61A) as monitored by the expression of HSV-1 UL30 gene and GFP signals (Figure 4C-4D). These data suggest that the enzymatic activity of USP18 is dispensable for DNA virus-triggered induction of type I IFNs and proinflammatory cytokines as well as cellular antiviral responses.

\section{USP18 recruits USP20 to deubiquitinate STING}

Because USP18 mediates deISGylation and interacts with STING, we examined whether USP18 regulates virus-triggered signaling through deISGylation of STING. Interestingly, however, STING was poorly ISGylated and neither USP 18 nor USP 18 (C64S) affected the ISGylation of STING even in the presence of IFN- $\beta$ (Supplementary information, Figure S5A). In addition, knockdown of ISG15 in Usp18 ${ }^{-/}$Ifnar $^{-/}$MEFs did not restore HSV-1-induced expression of Ifnb, Cxcll and Il6 (Supplementary information, Figure S5B), indicating that USP18 mediates antiviral signaling independently of deISGylation.

Although USP18 mediates deubiquitination of several substrates [46, 47], the DUB activity of USP18 is dispensable for regulation of antiviral signaling (Figure 4). Consistent with this notion, both human USP18 and USP18 (C64S) removed polyubiquitin chains from human STING, and mouse USP18 or USP18 (C61A) deubiquitinated mouse Sting (Figure 5A and Supplementary information, Figure S5C). Furthermore, neither overexpression of USP18 or USP18 (C64S) nor knockdown of USP18 affected the interaction between STING and RNF5, TRIM32, TBK1 or STING, indicating that USP18 might not be involved in ubiquitination or activation of STING (Supplementary information, Figure S6). We next examined whether USP18 recruited other DUBs for deubiquitination of STING. Of the DUBs interacting with STING, USP13, USP20, USP49 and USP50 interacted with USP18 in overexpression and coimmunoprecipitation assays, and USP20 and USP49 removed polyubiquitin chains from STING (Supplementary information, Figure S7A-S7B). We further found that the enzyme-inactive USP20 mutant (USP20 (C560/563S)) lost its ability to deubiquitinate STING (Figure 5B) and knockdown of USP20 substantially inhibited USP18-mediated deubiquitination of STING, whereas knockdown of USP18 impaired USP20-mediated deubiquitination of STING in HEK293 or HeLa cells (Figure 5C and Supplementary information, Figure S7C), indicating that USP18 and USP20 are mutually required for deubiquitination of STING.

We next investigated whether USP20 interacts with STING through USP18. As shown in Figure 5D, HSV-1 infection induced USP20-USP18-STING association in wild-type BMDCs and this association was completely abolished in Usp $18^{-/-}$BMDCs, indicating that USP18 is indispensable for the interaction between STING and USP20 after viral infection. Results from domain mapping analysis showed that USP20 interacts with the C-terminus (aa 151-372) of USP18 (Supplementary information, Figure S8A), while STING binds to the N-terminus (aa 1-150) of USP18 (Figure 1C). Interestingly, both USP18 and USP20 interact with the N-terminus of STING (aa 1-160) (Figure 1D and Supplementary information, Figure S8B). We reconstituted Usp $18^{-/-}$MEFs with different USP 18 truncates and examined HSV1- or cytoplasmic DNA-induced expression of type I IFNs and proinflammatory cytokines as well as STINGUSP20 association. The results showed that introduction of USP18 (51-372) (which interact with both USP20 and STING), but not USP18 (1-150) or USP18 (151-372), into Usp $18^{-/-}$cells restored the expression of downstream genes and STING-USP20 association after HSV-1 infection or transfection of various dsDNA ligands (Supplementary information, Figure S8C-S8D), supporting the notion that USP18 interacts with STING and recruits USP20 to STING through its N-terminal (aa 1-150) and C-terminal (aa 151-372) domains, respectively. Consistently, neither USP18 nor USP18 (C64S) deubiquitinated STING in vitro and USP20 but not USP20 (C560/563S) substantially deubiquitinated STING in the presence of USP18 or USP18 (C64S) (Figure 5E). In addition, re- 
A
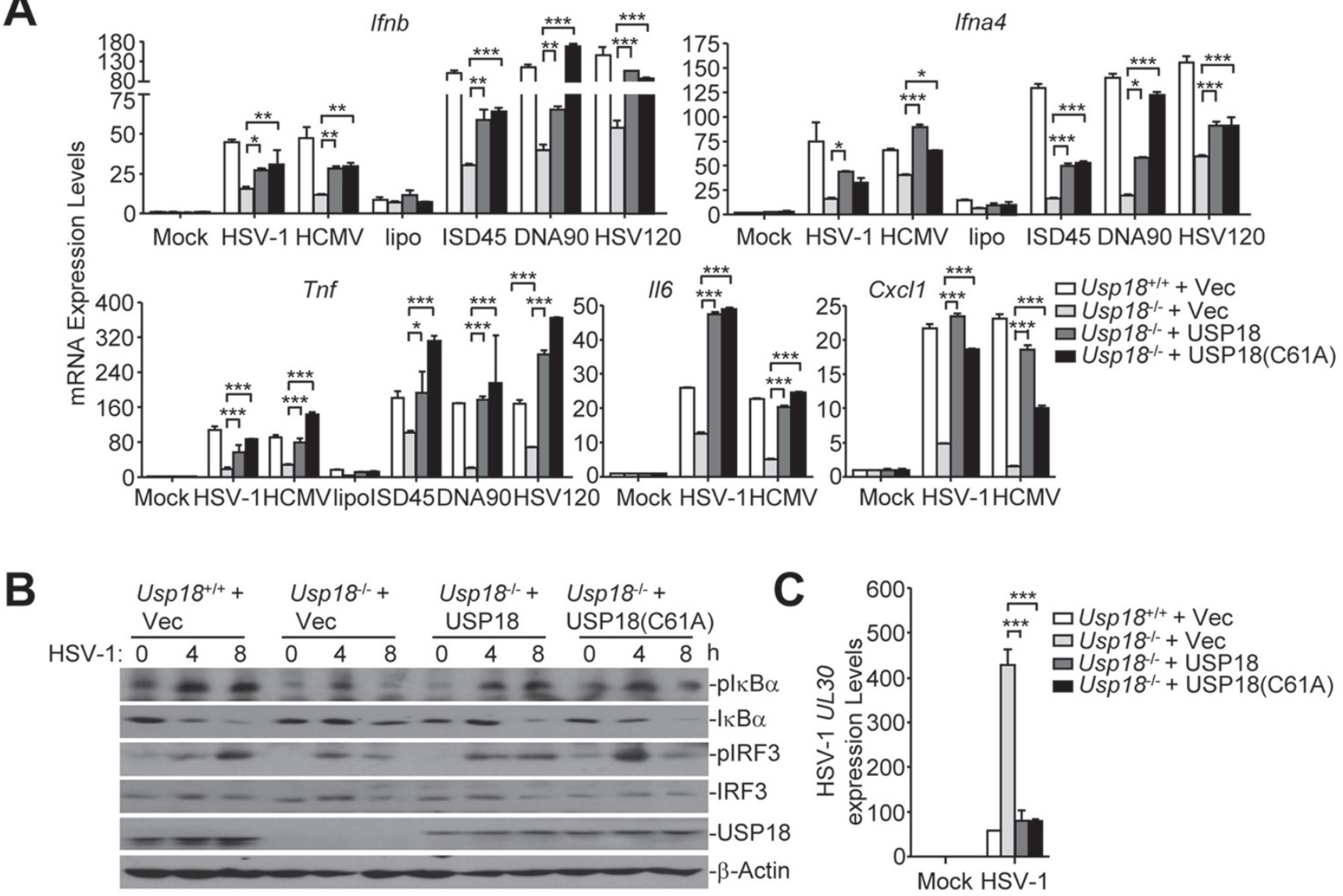

D

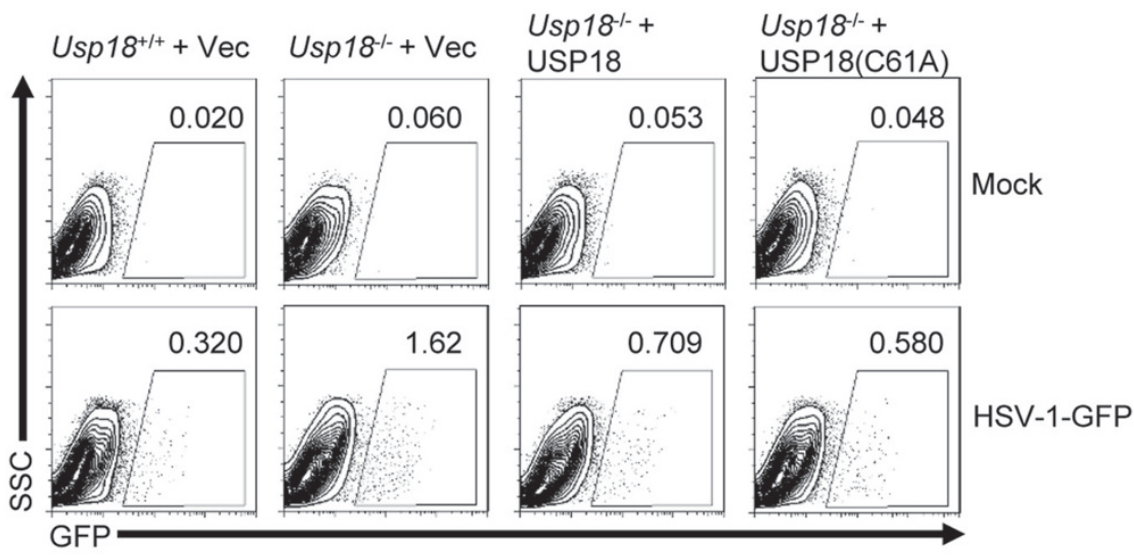

Figure 4 USP18 mediates antiviral signaling independently of its DUB activity. (A) qRT-PCR analysis of Ifnb, Ifna4, Tnf and

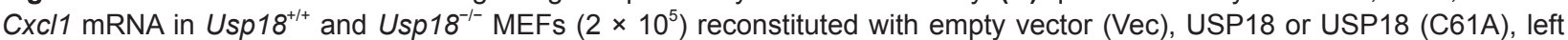
uninfected (Mock) or untransfected (Lipo), or infected with HSV-1, HCMV, SeV, EMCV or transfected with ISD45, DNA90 or HSV120 (3 $\mu \mathrm{g})$. (B) Immunoblot analysis of total and phosphorylated (p-) I $\mathrm{B} \alpha$ and IRF3, total USP18 and $\beta$-Actin in Usp18 ${ }^{+/+}$ and Usp $18^{-1-}$ MEFs $\left(1 \times 10^{6}\right)$ reconstituted with empty vector $(\mathrm{Vec})$, USP18 or USP18 $(\mathrm{C} 61 \mathrm{~A})$ and infected with HSV-1 for 4-8 h. (C) qRT-PCR analysis of HSV-1 UL30 mRNA in Usp18 $8^{+/+}$and Usp18 $8^{-/-}$MEFs $\left(2 \times 10^{5}\right)$ reconstituted with empty vector (Vec), USP18 or USP18 $($ C61A) infected with HSV-1 (MOI = 0.05) for $1 \mathrm{~h}$ followed by twice PBS wash and cultured with full medium for $24 \mathrm{~h}$. (D) Flow cytometry analysis of the replication of GFP-tagged HSV-1 in Usp $18^{+/+}$and Usp $18^{-/-} \mathrm{MEFs}\left(2 \times 10^{5}\right)$ reconstituted with empty vector (Vec), USP18 or USP18 (C61A) and infected with HSV-1-GFP (MOI $=0.05)$ for 24 h. ${ }^{*} P<0.05,{ }^{* *} P$ $<0.01$ and ${ }^{* *} P<0.001$ (analysis of two-way ANOVA followed by Bonferroni post-test). Data are representative of three (A) or two (B-D) independent experiments (mean \pm SD in $\mathbf{A}$ and $\mathbf{C}$ ). 
A

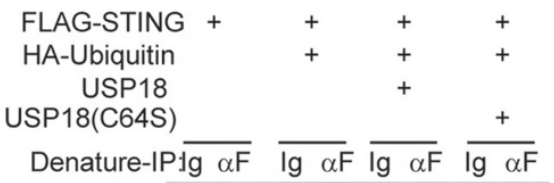

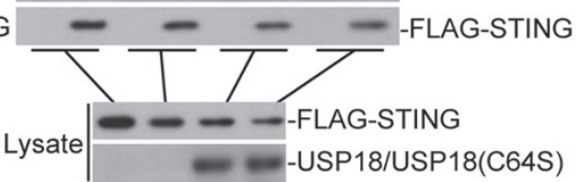

B

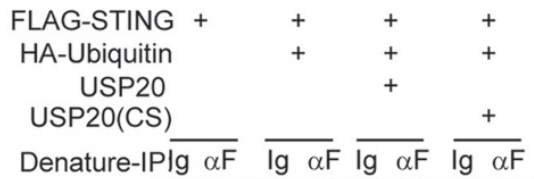

IB: $\alpha \mathrm{HA}$

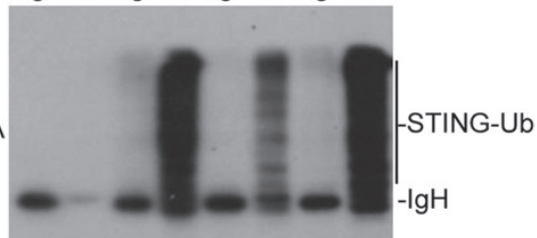

IB: $\alpha$ FLAG

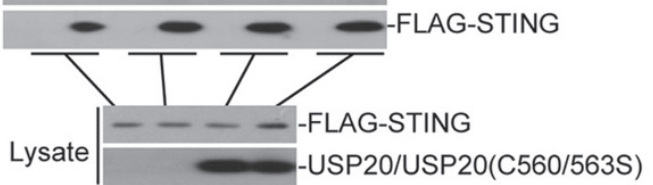

C

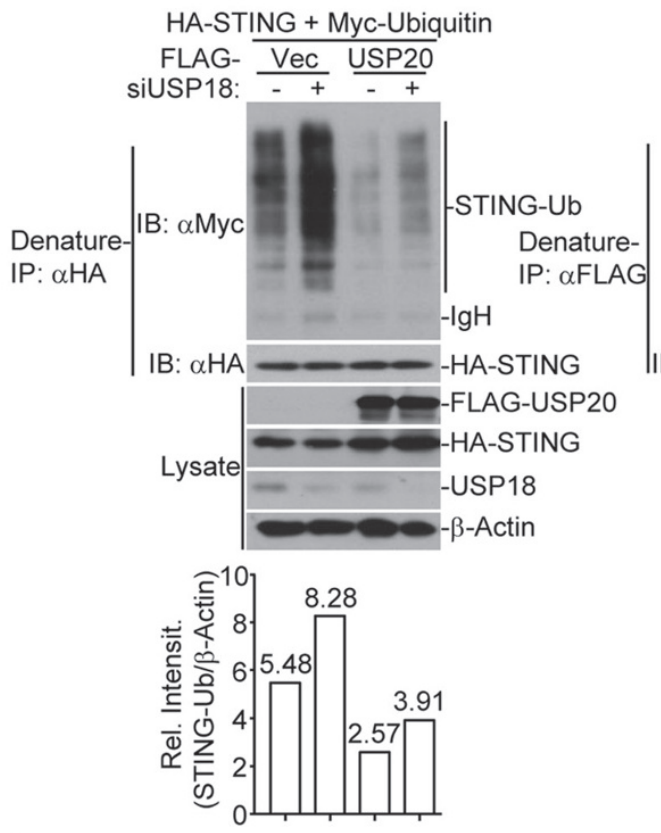

FLAG-STING + Myc-Ubiquitin pCMV-
sec
$\frac{\text { USPP18 }}{-++}$

IB: $\alpha M y c$

D

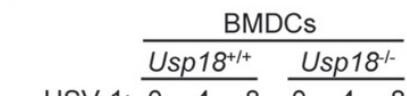

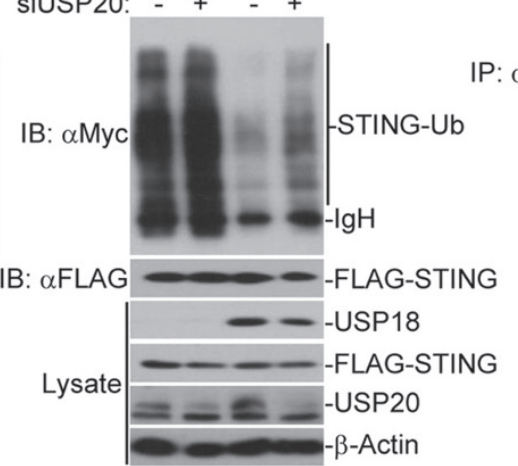

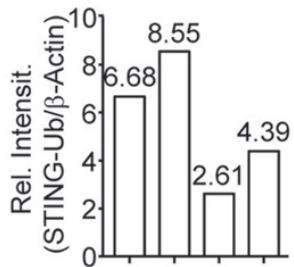

E

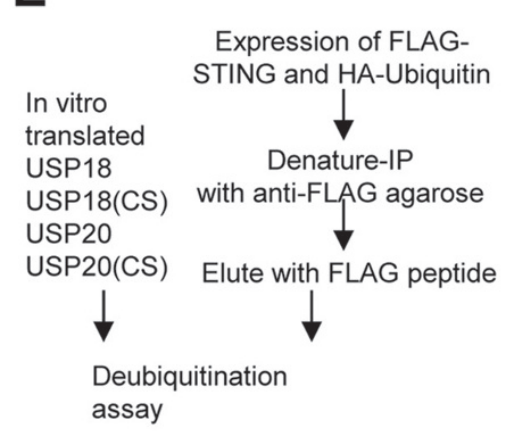

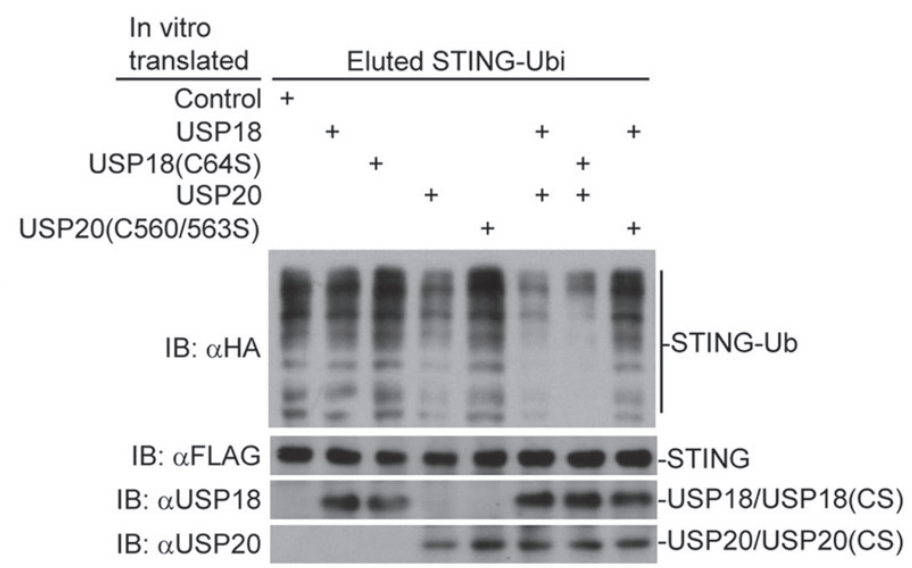


constitution of Usp18 $8^{-/-}$MEFs with full-length USP18 or USP18 (51-372), but not USP18 (1-150) or USP18 (151-372), impaired HSV-1-triggered ubiquitination of STING (Supplementary information, Figure S8E). These data together suggest that USP20 is recruited to STING through USP18 and deubiquitinates STING after HSV-1 infection.

\section{USP20 is involved in HSV-1-induced signaling}

We next examined the role of USP20 in regulating virus-triggered signaling and found that HSV-1- but not SeV-induced expression of IFNB, IL6, IP10 and CCL5 was significantly impaired in THP-1 cells by knockdown of USP20 (Figure 6A). In addition, the expression levels of IFNB, IL6, IP10 and CCL5 were significantly reduced in USP20-knockdown THP-1 cells after transfection of various DNA ligands (Figure 6A). Consistently, knockdown of USP20 inhibited HSV-1- but not SeV-induced phosphorylation of IRF3 or I $\mathrm{BB} \alpha$ (Figure 6B). The GFP percentages and the expression level of HSV-1 UL30 gene were increased in THP-1 cells transfected with siUSP20 compared with the control after HSV-1-GFP and HSV-1 infection, respectively (Figure 6C-6D). These data together suggest that USP20 positively regulates DNA virus-induced antiviral responses.

\section{USP20 deconjugates K33/48-linked ubiquitination of and stabilizes STING}

Because USP20 removes ubiquitin modification from STING and is required for DNA virus-triggered signaling, we investigated the type(s) of polyubiquitin chains of STING that are deconjugated by USP20. We cotransfected FLAG-STING with HA-tagged ubiquitin, or ubiquitin mutants retaining a single lysine (KO) into HEK293 cells in the presence or absence of USP20 followed by deubiquitination assays (Figure 7A). The results showed that USP20 mainly removed K33- or K48-linked ubiquitination of STING, as USP20 did not remove K33/48Rlinked polyubiquitin chains (in which lysines 33 and 48 of ubiquitin were simultaneously mutated into arginine) from STING (Figure 7B). In contrast, USP18 overexpression still led to partial removal of K33/48R-linked polyubiquitin chains (although to a less extent compared with wild-type ubiquitination) from STING (Supplementary information, Figure S9A), indicating that USP18 might recruit other $\operatorname{DUB}(\mathrm{s})$ to deconjugate other types of ubiquitin chains from STING. In addition, we found that USP20 deconjugated K48- but not K27-linked polyubiquitin chains from STING in the presence of USP18 or USP18 (C64S) in vitro (Supplementary information, Figure S9B). Although USP20 (at high dosages) alone deubiquitinated STING, a low dose of USP20 efficiently removed K48-linked polyubiquitin chains from STING in vitro in the presence of USP18 (Figure 5E and Supplementary information, Figure S9C). These data together suggest that USP18 recruits and facilitates USP20 to deconjugate K48-linked polyubiquitin chains from STING.

Consistent with these observations, HSV-1-induced K48-linked ubiquitination of STING was substantially enhanced in Usp $18^{-/-}$BMDCs, USP20-knockdown THP1 cells, or USP18 and USP20 double-knockdown THP1 cells compared with the controls (Figure 7C-7D and Supplementary information, Figure S10). In contrast, HSV-1-induced ubiquitination of TRAF6 was not potentiated in Usp $18^{-/-}$MEFs compared with the wildtype MEFs, although USP18 interacted with TRAF6 in an overexpression system (Supplementary information, Figure S11A-S11B). In addition, compared with the controls, Usp $18^{-/-}$BMDCs and USP20-knockdown THP-1 cells showed reduction in STING protein levels

Figure 5 USP18 recruits USP20 to deubiquitinate STING. (A) Immunoblot of HEK293 cells that were transfected to express FLAG-STING, HA-Ubiquitin, along with vector, USP18 or USP18 (C64S), lysed and immunoprecipitated with control IgG or anti-FLAG. Cell lysate was analyzed by immunoblot with anti-FLAG or anti-USP18. (B) Immunoblot of HEK293 cells that were transfected to express FLAG-STING, HA-Ubiquitin, along with vector, USP20 or USP20 (C560/563S), lysed and immunoprecipitated with control IgG or anti-FLAG. Cell lysate was analyzed by immunoblot with anti-FLAG or anti-USP20. (C) Immunoblot of HEK293 cells transfected with a control siRNA, siUSP18 (left panels) or siUSP20 (right panels) for $12 \mathrm{~h}$ followed by transfection of HA-STING and Myc-Ubiquitin, along with FLAG-USP20 (left panels) or pCMV-USP18 (right panels) for $24 \mathrm{~h}$, lysed and immunoprecipitated with anti-HA (left) or anti-FLAG (right). Cell lysate was analyzed by immunoblot with anti-FLAG, anti-HA, anti-USP20, anti-USP18 or anti- $\beta$-Actin. The intensities of ubiquitin-modified STING were normalized to $\beta$-Actin (graphs below). (D) Immunoblot of Usp18 ${ }^{+/+}$and Usp $18^{-/-}$BMDCs infected with HSV-1 for 4-8 h, lysed and immunoprecipitated with anti-STING followed by immunoblot analysis with antibodies to the indicated proteins. (E) In vitro deubiquitination analysis of ubiquitin-modified STING eluted from the denature immunoprecipitates (anti-FLAG) from HEK293 cells transfected with FLAG-STING and HA-ubiquitin with FLAG peptide followed by incubation with in vitro generated USP18, USP18(C64S), USP20, USP20(C560/563S) by an in vitro transcription and translation kit. The mixtures were analyzed by immunoblot analysis with antibodies against HA, FLAG, USP18 or USP20. Data are three (A-C) or two (D-E) independent experiments. 

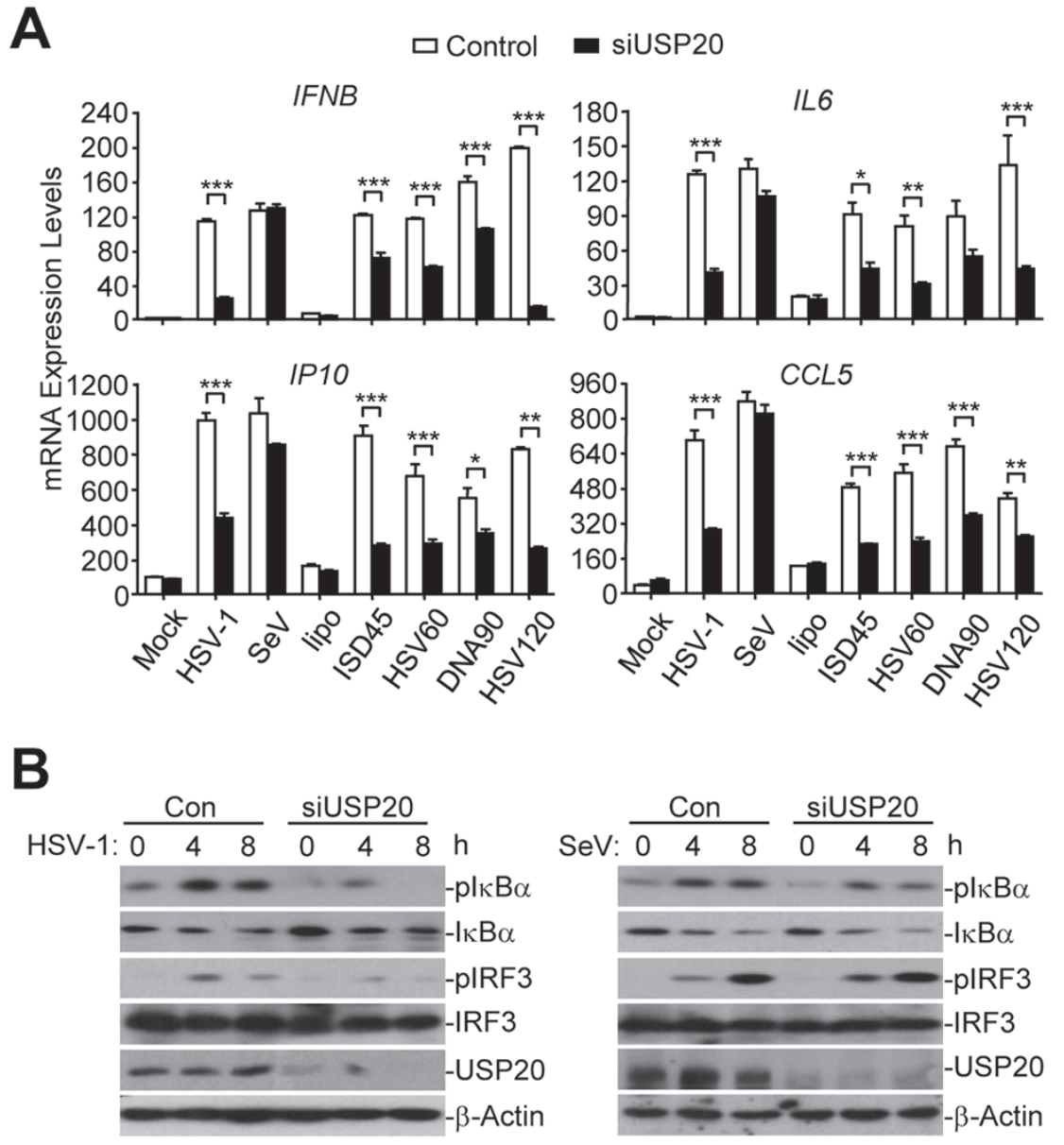

C
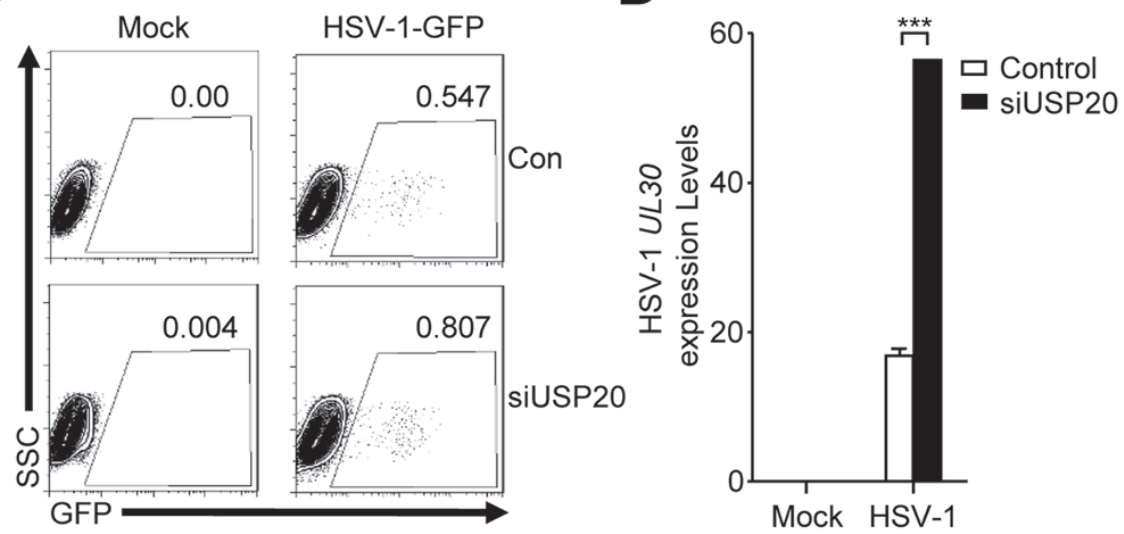

Figure 6 USP20 is involved in HSV-1-triggered signaling. (A) qRT-PCR analysis of IFNB, IL6, IP-10 and CCL5 mRNA in THP-1 cells $\left(5 \times 10^{5}\right)$ transfected with control siRNA (Control) or siRNA targeting USP20 (siUSP20) for $36 \mathrm{~h}$ left uninfected or untransfected, or infected with HSV-1, SeV or transfected with ISD45, HSV60, DNA90 and HSV120 (3 $\mu$ g). (B) Immunoblot of total and phosphorylated ( $\mathrm{p}-\mathrm{I}_{\kappa \mathrm{B}} \alpha$ and IRF3, total USP20 and $\beta$-Actin in ThP-1 cells $\left(5 \times 10^{6}\right)$ transfected with control siRNA (Con) or siUSP20 followed by HSV-1 infection for 0-8 h. (C) Flow cytometry analysis of GFP-tagged HSV-1 (MOI = 0.1) in THP-1 cells $\left(5 \times 10^{5}\right)$ transfected with control siRNA (Con) or siUSP20 followed by infection with HSV-1-GFP for 24 h. (D) qRT-PCR analysis of HSV-1 UL30 mRNA in THP-1 cells $\left(5 \times 10^{5}\right)$ transfected with control siRNA (Control) or siUSP20 infected with HSV-1 $(\mathrm{MOI}=0.1)$ for $1 \mathrm{~h}$ followed by PBS wash twice and cultured with full medium for $24 \mathrm{~h} .{ }^{*} P<0.05$, ${ }^{* *} P<0.01$ and ${ }^{* * *} P<0.001$ (analysis of two-way ANOVA followed by Bonferroni post-test). Data are representative of three (A, B) or two (C, D) independent experiments (mean \pm SD in $\mathbf{A}$ and $\mathbf{D}$ ). 
after HSV-1 infection or cytoplasmic DNA transfection. Such reduction was blocked by the proteasome inhibitor MG132 in Usp $18^{-/-}$BMDCs or in USP20-knockdown THP-1 cells but not by the autophagy inhibitor 3MA in USP20-knockdown THP-1 cells (Figure 7E-7H), indicating that USP18 and USP20 prevent STING protein from proteasome-dependent degradation. Consistently, the mRNA levels of STING were not affected by USP18 deficiency or by USP20 knockdown in the presence or absence of HSV-1 infection (Supplementary information, Figure S11C). Consistent with these observations, reconstitution of Usp $18^{-/-}$MEFs with STING restored HSV-1- or HCMV-induced expression of type I IFNs and proinflammatory cytokines as well as cellular antiviral responses (Supplementary information, Figure S11DS11E). Taken together, these data suggest that USP18 recruits USP20 to remove K48-linked ubiquitination of STING and thereby maintains stability of STING to promote expression of type I IFNs and proinflammatory cytokines after HSV-1 infection.

\section{Discussion}

Ubiquitination of STING plays critical roles in virus-triggered signaling. In this study, we have identified USP18 as a STING-interacting DUB that recruits USP20 to remove K33- and K48-linked ubiquitin chains from STING and thereby prevents proteasome-dependent degradation of STING after DNA virus infection (Supplementary information, Figure S12). In support of this conclusion, we observed that (i) USP18 deficiency or knockdown of USP20 impaired HSV-1- or cytoplasmic DNA-induced activation of IRF3 and NF- $\kappa \mathrm{B}$ and subsequent expression of type I IFNs and proinflammatory cytokines, (ii) knockdown of USP20 or USP18 deficiency resulted in enhanced replication of HSV-1 and USP18-deficient mice were more susceptible to HSV-1 infection compared with the wild-type littermates, (iii) USP18 and USP20 were mutually required to deubiquitinate STING, (iv) USP20 removed K33O-, or K48O- but not K33/48R-linked polyubiquitin chains from STING, and (v) HSV-1- or cytoplasmic DNA-induced degradation of STING was blocked by the proteasome inhibitor MG132 in Usp18 ${ }^{-/-}$MEFs and USP20-knockdown THP1 cells. These findings advance our understanding of the complicated regulatory mechanisms of virus-triggered signaling.

It has been demonstrated that USP18 negatively regulates type I IFN-triggered signaling by competing for JAK1 association with IFNAR2 in a manner independent of its enzymatic activity [43]. Therefore, USP 18 deficiency inhibited viral replication after type I IFN treatment in vitro. However, the Usp $18^{-/-}$mice have been reported to exhibit resistance, susceptibility or normal response to viral infection compared with the wild-type controls when challenged by LCMV or VSV in vivo [45, 48, 49]. Indeed, USP18-deficient DCs fail to present the viral antigens efficiently to T cells after VSV infection but are fully capable of presenting the viral antigens to $T$ cells in the presence of IFN- $\alpha$ after UV-inactivated VSV infection [49], indicating that Usp $18^{-/-}$cells might be defective in type I IFN production after viral infection and thus incapable of presenting antigen to modulate the adaptive immunity. In our study, we found that USP 18 deficiency led to impaired activation of IRF3 and NF$\kappa \mathrm{B}$ and induction of type I IFNs and potentiated HSV-1 replication, suggesting an indispensable role of USP 18 in innate antiviral immunity. Because USP18 deficiency results in impaired innate immune responses after viral infection, it is conceivable that the viral specific $\mathrm{T}$ cell and humoral responses are severely compromised in Usp $18^{-/-}$ mice compared with the wild-type controls after viral infection [49]. More recently, it has been demonstrated that USP18 deficiency in human results in the severe pseudo-TORCH syndrome which is a clinical phenotype related with in utero exposure to infection but with a noninfectious etiology $[50,51]$. Cells and brain tissues from the USP18-dificient pseudo-TORCH syndrome patients exhibit hypersensitivity to type I IFNs, indicating that the major function of USP18-mediated negative regulation of type I IFN signaling is to restrict excessive immune responses rather than to promote innate antiviral responses. Taken together, our results and others collectively suggest a step-wise and protective role of USP 18 against viral infection, i.e., promoting virus-triggered type I IFN induction and cellular antiviral responses and subsequently restricting excessive harmful immune responses.

USP18 was originally identified as a DUB and later characterized as a deISGylation enzyme [39, 40, 52]. Various studies have demonstrated that USP18 deletion results in increased ISGylation after viral infection [42, $48,53]$. However, deletion of ISG15 or impairment of ISGylation in Usp $18^{-/-}$background had no effect on viral replication or type I IFN-triggered signaling in vitro [44, 45]. In our study, we found that knockdown of ISG15 did not affect HSV-1-induced expression of type I IFNs and proinflammatory cytokines in $U_{s p} 18^{+/ /}$Ifnar $^{-/-}$or Usp $18^{-/-}$Ifnar $1^{-/-}$MEFs, indicating that USP18 functions independently of deISGylation in the innate antiviral signaling. In addition, reconstitution of wild-type USP18 or USP18 (C61A) into Usp $18^{-/-}$MEFs restored HSV-1induced activation of IRF3 and NF- $\kappa$ B and expression of downstream genes and inhibited HSV-1 replication, 
A

FLAG-STING

HA-Ubi: WT K6O K110 K270 K290 K33O K48O K630

pCMV-USP20: $\frac{\mathrm{W}}{-+} \frac{\mathrm{K} 6 \mathrm{O}}{-+} \frac{\mathrm{K} 110}{-+} \frac{\mathrm{K} 270}{+} \frac{\mathrm{K} 290}{-+} \frac{\mathrm{K} 330}{-+} \frac{\mathrm{K} 480}{-+} \frac{\mathrm{K} 63 \mathrm{O}}{-+}$

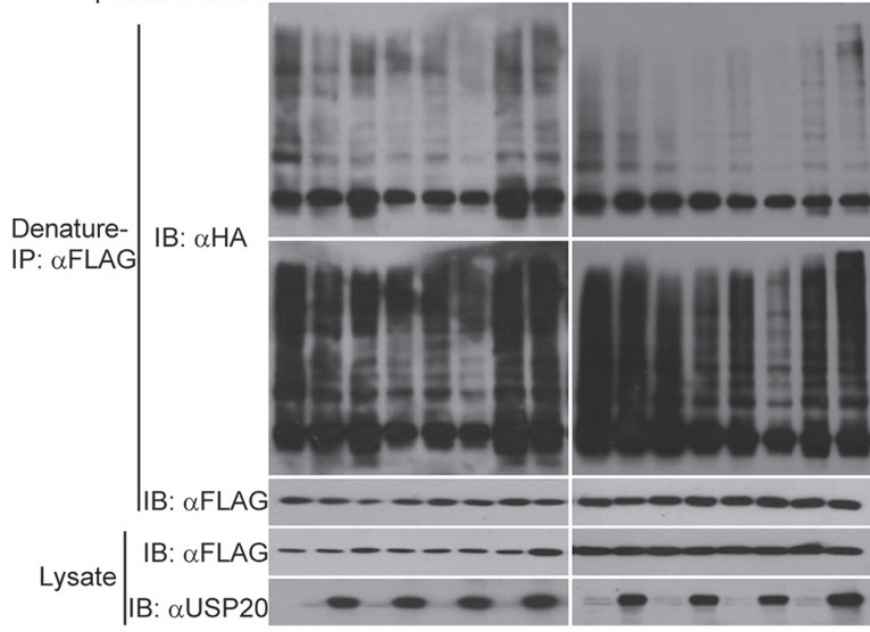

B

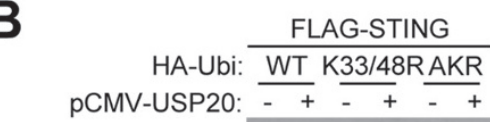

STING-Ubi

Weak exp.

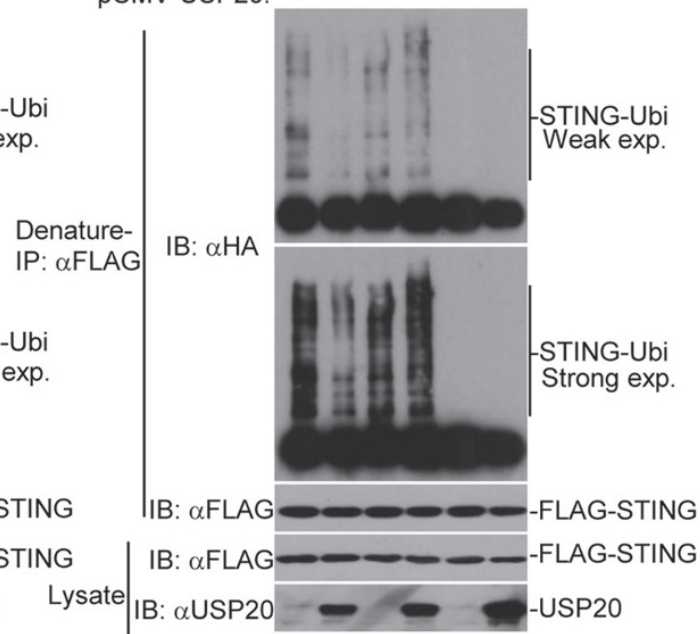

C $\frac{\text { BMDCs }}{\frac{U s p 18^{+/+}}{048} \frac{U s p 18^{-1 /}}{048}}$

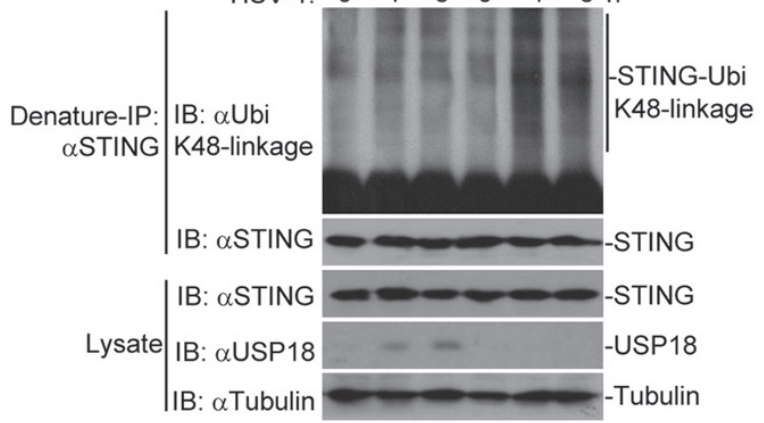

D

-FLAG-STING

-FLAG-STING

-USP20
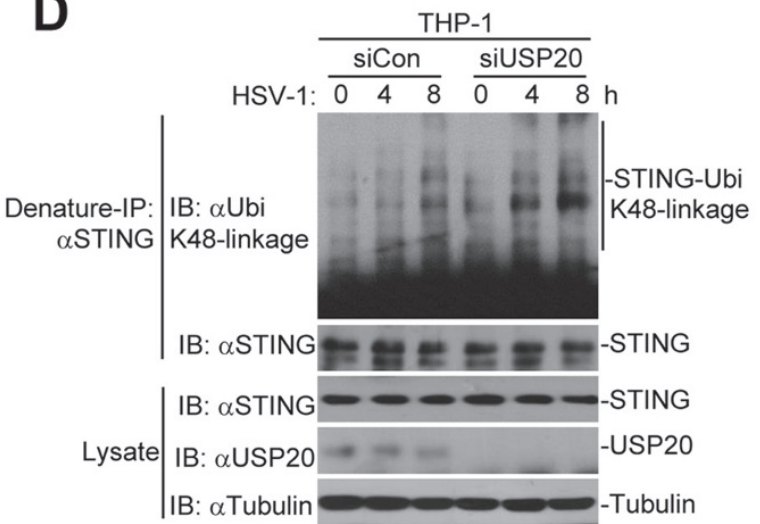

E

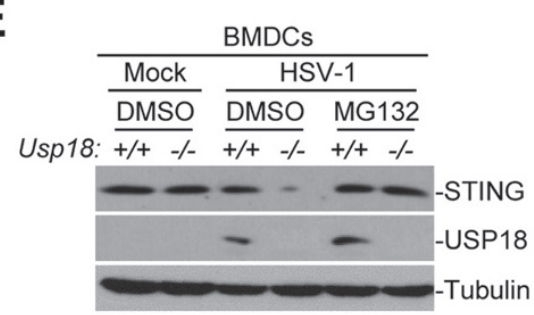

\section{G}

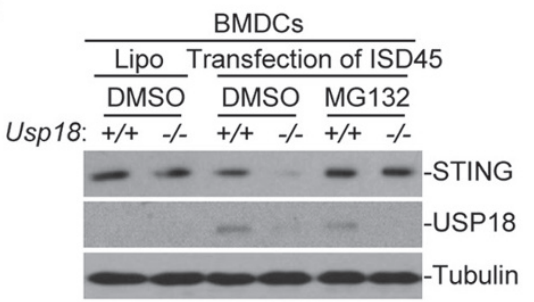

F

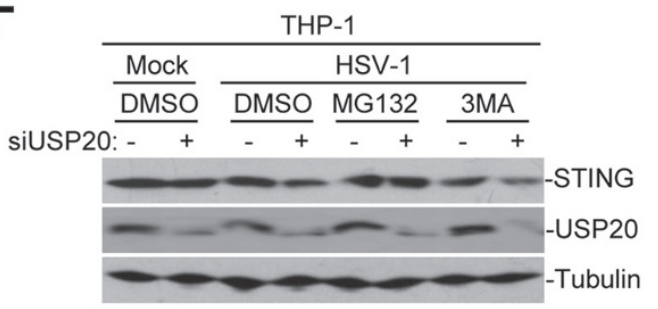

H

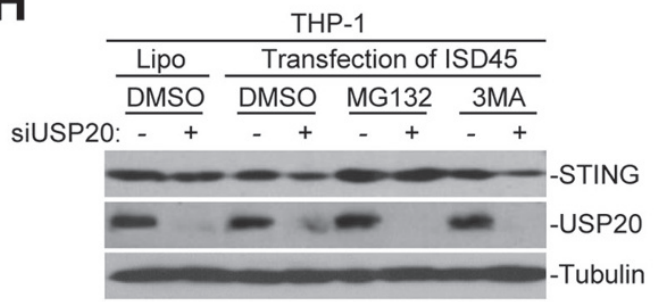


indicating that USP 18 mediates the innate antiviral signaling independently of its enzymatic activity. Consistently, a recent study has demonstrated that VSV- or VACV-induced phosphorylation of IRF3 is comparable between wild-type and USP $18^{\mathrm{C} 61 \mathrm{~A}}$ cells [42]. Interestingly, the USP $18^{\mathrm{C} 61 \mathrm{~A}}$ mice exhibit resistance to influenza $\mathrm{B}$ virus infection, which is compromised by deletion of ISG15, whereas type I IFN-triggered phosphorylation of STAT1 is comparable between wild-type and USP $18^{\mathrm{C} 61 \mathrm{~A}}$ cells [42], indicating that USP18-mediated deISGylation rather than regulation of type I IFN signaling is involved in antiviral responses. Therefore, it is likely that USP18 promotes and inhibits antiviral immune responses through deISGylation-independent and dependent manners, respectively. How the deISGylation-dependent and -independent roles of USP18 are entangled in antiviral immunity requires further investigations. Nonetheless, our results have clearly demonstrated that USP18 positively regulates virus-triggered induction of type I IFNs and proinflammatory cytokines and cellular antiviral responses in a deISGylation-independent manner.

USP18 has been demonstrated to deubiquitinate the TAK1/TABs complex downstream of TCR signaling, thereby inhibiting NF- $\kappa B$ and NFAT activation [46]. However, the DUB activity of USP18 is not required for deubiquitinating STING or for mediating virus-triggered induction of type I IFNs and proinflammatory cytokines. Instead, USP18 recruits USP20 to deconjugate ubiquitin chains from STING. First, USP18 or USP18 (C64S) did not affect interaction between STING and RNF5, an E3 ligase mediating K48-linked ubiquitination of STING [28]. Second, knockdown of USP18 impaired USP20-mediated deubiquitination of STING and knockdown of USP20 inhibited deconjugation of STING by USP18. Third, USP20 interacted with STING upon HSV-1 infection, which was abolished in Usp18 $8^{-/-}$ cells. Fourth, USP18 or USP18 (C64S) alone did not remove polyubiquitin chains from STING and USP20 exhibited weak deubiquitination activity for STING in vitro, whereas USP18 or USP18 (C64S) plus USP20 but not USP20(C560/563S) substantially catalyzed deubiquitination of STING in vitro, indicating that USP20 is the authentic DUB for deubiquitination of STING in the presence of USP18. It is not surprising because USP18 regulates TLR-triggered signaling partially through sequestration of IKK $\gamma$ from K63-linked ubiquitination, which is independent of its DUB activity [47]. Thus, USP18 may function in at least three different manners in distinct signaling pathways, including deubiquitination, deISGylation and a manner independent of deubiquitination and deISGylation.

It is generally accepted that the cGAS-STING pathway is responsible for recognition of cytoplasmic DNA and defense against DNA viruses [54]. USP18 deficiency or knockdown of USP20 resulted in enhanced K48linked ubiquitination of STING and accelerated degradation of STING, decreased activation of IRF3 and NF- $\mathrm{kB}$ and reduced induction of downstream genes after HSV1 infection or transfection of DNA ligands. Although USP 18 interacted with TRAF6 in an overexpression system, the ubiquitination of TRAF6 was not potentiated in Usp $18^{-/-}$MEFs compared with wild-type MEFs after HSV-1 infection, indicating that USP18 did not deubiquitinate TRAF6. In addition, reconstitution of STING into Usp $18^{-/-}$MEFs restored HSV-1- or HCMV-induced expression of type I IFNs and proinflammatory cytokines, indicating that USP18 positively regulates DNA virus-triggered signaling primarily through promoting the deubiquitination and stabilization of STING.

Several studies have suggested that STING also mediates RNA virus-triggered signaling. STING is an important adaptor protein in RNA virus-triggered signaling in HEK293 and HeLa cells but not in THP-1 cells [19, 55]. STING-deficient MEFs or BMDCs are sensitive to infection with VSV and produced decreased levels of type I IFNs after $\mathrm{SeV}$ or West Nile virus (WNV) infec-

Figure 7 USP20 deconjuagates K33/48-linked ubiquitination of and stabilizes STING. (A) Immunoblot of HEK293 cells that were transfected to express FLAG-STING, pCMV-USP20, HA-tagged Ubi (WT) and various mutants, lysed and immunoprecipitated with anti-FLAG. Cell lysate was analyzed by immunoblot with anti-FLAG or anti-USP20. (B) Immunoblot of HEK293 cells that were transfected to express FLAG-STING, pCMV-USP20, HA-Ubi (WT), K33/48R mutant of HA-Ubi or AKR, Iysed and immunoprecipitated with anti-FLAG. Cell lysate was analyzed by immunoblot with anti-FLAG anti-USP20. (C-D) Immunoblot of Usp18 ${ }^{+/+}$and Usp18 $8^{-/-}$BMDCs $\left(5 \times 10^{6}\right)\left(\right.$ C) or THP-1 cells $\left(5 \times 10^{6}\right)$ transfected with control siRNA (siCon) or siUSP20 (D) infected with HSV-1 for 0-8 $\mathrm{h}$ in the presence of MG132 (10 $\mu \mathrm{M})$, lysed and immunoprecipitated with anti-STING in the presence of NEM $(20 \mathrm{mM})$ followed by immunoblot analysis with antibodies against the indicated proteins. (E, F) Immunoblot analysis of STING, Tubulin, USP18 or USP20 in Usp18 ${ }^{+/+}$and Usp $18^{-/-}$BMDCs $\left(2 \times 10^{6}\right)(E)$ or in THP-1 cells $\left(1 \times 10^{6}\right)$ that were transfected with control siRNA (-) or siUSP20 (+) (F) followed by HSV-1 infection in the presence or absence of MG132 or 3 MA for 6 h. (G, H) Immunoblot analysis of STING, Tubulin, USP18 or USP20 in Usp $18^{+/+}$and Usp $18^{-/-}$BMDCs $\left(2 \times 10^{6}\right)$ (G) or in THP-1 cells $\left(1 \times 10^{6}\right)$ that were transfected with control siRNA $(-)$ or siUSP20 $(+)(H)$ followed by transfection with ISD45 in the presence or absence of MG132 or 3MA for $3 \mathrm{~h}$. Data are representative of three independent experiments. 
tion $[20,56]$. Moreover, STING-deficient mice are more susceptible to lethal VSV and WNV inoculation. A recent study suggests that membrane fusion of enveloped RNA viruses stimulates type I IFN induction through STING-dependent but cGAS-independent signaling [57]. In our study, we found that USP18 deficiency resulted in partial impairment of type I IFN induction in response to $\mathrm{SeV}$ infection. Interestingly, whereas USP20 did not remove K33/48R-linked polyubiquitin chains from STING and was involved in HSV-1- but not SeV-triggered signaling, USP18 promoted the deconjugation of K33/48Rlinked ubiquitination of STING and USP18 deficiency impaired RNA virus-triggered signaling. A simplest explanation for this phenomenon is that USP18 regulates RNA virus-triggered signaling through promoting the deconjugation of ubiquitin chains other than the K33- or K48-linked type from STING. In this context, we have observed that $\mathrm{SeV}$ induced ubiquitination of STING in various human and mouse cells [28]. Alternatively, USP18 might target other molecules involved in RNA virus-triggered signaling, thereby promoting innate antiviral responses against RNA viruses. USP20 has been implicated in DNA damage repair signaling by deubiquitinating Claspin $[37,38]$. We found that USP20 mainly deconjugated K33- and K48-linked ubiquitination of STING and prevented proteasome-dependent degradation of STING. How the K33-linked ubiquitination of STING is mediated and whether USP18 recruits other DUBs to deconjugate polyubiquitin chains of other linkage types from STING require further study.

In conclusion, our findings have characterized new roles of USP18 and USP20 in mediating virus-triggered induction of type I IFNs and innate immune responses against viral infection. Importantly, USP18-mediated antiviral signaling is independent of its enzymatic activity but depends on the recruitment of USP20 for deconjugation of K33- and K48-linked ubiquitination of STING. Thus, targeting the non-enzymatic function of USP18 or the DUB activity of USP20 may benefit treatment of infectious diseases in the future.

\section{Materials and Methods}

Mice

Usp $18^{+/-}$mice were previously described [58] and crossed to obtain Usp $18^{++\mid}$and Usp $18^{-/-}$mice. Ifnar $1^{-/-}$mice were purchased from The Jackson Laboratory and crossed with Usp $18^{-/-}$mice. The Ifnar $1^{-/} U s p 18^{+/-}$were crossed to obtain Ifnar $1^{-/} U s p 18^{+/+}$and Ifnarl $1^{-1}$ Usp $18^{-1-}$ mice for experiments. All mice were housed in the specific pathogen-free animal facility at Wuhan University and all animal experiments were in accordance with protocols approved by the Institutional Animal Care and Use Committee of Wuhan University.

\section{Reagents, antibodies and constructs}

ISD45, HSV60, DNA90, and HSV120 were described previously [59]. MG132 and 3MA were purchased from Sigma. Mouse control IgG (Santa Cruz Biotechnology, sc-2025) and rabbit control IgG (Millipore, 12-370), HRP-conjugated goat-anti-mouse or rabbit IgG (Thermo Scientific, PA1-86717 and SA1-9510), mouse anti-FLAG (Sungene, KM8002), anti- $\beta$-Actin (KM9001), anti-Tubulin (F0601), anti-HA (COVANCE, MMS-101R), anti-pIкB $\alpha$ (9246L), anti-Ubiquitin (sc-8017), anti-ubiquitin K48-specific linkage (Millipore, NG1827433), anti-IRF3 (sc-9082), anti-IкB $\alpha$ (sc-371), anti-p-IRF3 (Cell Singling Technologies, 4947S), anti-USP18 (sc-50019), anti-USP20 (Abcam, A301-189A-T-2) and anti-STING (13647S) were purchased from the indicated manufactures. The ISRE and IFN- $\beta$ promoter luciferase reporter constructs, mammalian expression plasmids for STING, STING truncations, TRAF6, p65, MAVS, TBK1, IRF3, RIG-I, ubiquitin and ubiquitin mutations were previously described [19, 28, 59]. The construct encoding cGAS was a kind gift from James Zhijian Chen (The University of Texas Southwest Medical Center). Mammalian expression plasmids for wild-type and mutant USP18 and USP20 were constructed by standard molecular biology techniques. Staining antibodies against CD3, CD4, CD8, CD19, CD25, CD44, CD62L, CD11c, CD11b, B220 and F4/80 were purchased from Biolegend or Sungene. Flow cytometry analysis was performed with a Beckman COOLTER CyAn ADP system.

\section{qRT-PCR and ELISA}

Total RNA was extracted from cells using TRIzol (Invitrogen), and the first-strand cDNA was reversed-transcribed with All-inOne cDNA Synthesis SuperMix (Biotool). Gene expression was examined with a Bio-Rad CFX Connect system by a fast two-step amplification program with $2 \times$ SYBR Green Fast qRT-PCR Master Mix (Biotool). The value obtained for each gene was normalized to that of the gene encoding $\beta$-actin. Gene-specific primers have been described previously [60] or listed in Supplementary information, Table S1. The ELISA kits for IFN- $\alpha$ (Life Technologies), Cxcl1 and IL-6 (Biolgend) were used to detect the indicated cytokines in the sera.

\section{Co-immunoprecipitation and immunoblot analysis}

The experiments were performed as previously described [19, $28,60]$. In brief, cells were lysed in Nonidet P-40 lysis buffer containing $150 \mathrm{mM} \mathrm{NaCl}, 1 \mathrm{mM}$ EDTA, $1 \%$ Nonidet P-40, and 1\% protease and phosphatase inhibitor cocktail (Biotool). Cell lysates were subjected to SDS-PAGE and immunoblot analysis was performed with the appropriate antibodies. For immunoprecipitation assays, the lysates were immunoprecipitated with $\operatorname{IgG}$ or the appropriate antibodies, and the precipitants were washed three times with lysis buffer containing $500 \mathrm{mM} \mathrm{NaCl}$, followed by immunoblot analysis.

\section{In vivo and in vitro deubiquitination assays}

These experiments were performed as previously described [61]. For deubiquitination assays in cells, cells were lysed with the lysis buffer $(100 \mu \mathrm{l})$ and the supernatants were denatured at 95 ${ }^{\circ} \mathrm{C}$ for $5 \mathrm{~min}$ in the presence of $1 \%$ SDS by lysates. The denatured lysates were diluted with lysis buffer until the concentration of SDS was reduced $<0.1 \%$ followed by immunoprecipitation (denature-IP) with the indicated antibodies. The immunoprecipitants 
were subject to immunoblot analysis with anti-ubiquitin, or antiK48-linked ubiquitin chains. For in vitro deubiquitination, FLAGtagged STING and HA-tagged ubiquitin or ubiquitin mutants were cotransfected into HEK 293 cells. Denature-IP was performed and the precipitants were eluted by $3 \times$ FLAG peptide (sigma) to obtain ubiquitin-modified STING. USP18, USP18 (C64S), USP20 and USP20 (C560/563S) were obtained by a TNT in vitro transcription/translation kit (Promega). The ubiquitinated STING were incubated with in vitro synthesized proteins at $37^{\circ} \mathrm{C}$ for $2 \mathrm{~h}$ followed by overnight incubation at $16{ }^{\circ} \mathrm{C}$ in the presence of $1 \mu \mathrm{M}$ ATP. The mixture was analyzed by immunoblot with the indicated antibodies.

\section{Cell Culture}

Mouse embryonic fibroblasts were prepared from E14.5 embryos. Bone marrow cells were isolated from mouse femur. The cells were cultured in DMEM containing 20\% fetal bovine serum, $1 \%$ streptomycin and penicillin, and $10 \mu \mathrm{M} \beta$-mercaptoethanol, with M-CSF (10 ng/ml, Peprotech) for BMDM differentiation or GMCSF (20 ng/ml, Peprotech) for BMDC differentiation. For preparation of $\mathrm{pDCs}$, bone marrow cells were cultured in RPMI medium containing 10\% FBS, 1\% streptomycin-penicillin and recombinant mouse Flt3L (20 ng/ml, Peprotech). The medium was changed every 3 days. On day 7, cells were used for subsequent analysis. Specifically, the CD11c magnetic beads (Stem Cell) were used to select the $\mathrm{CD} 11 \mathrm{c}^{+}$BMDCs for subsequent analysis. Primary mouse lung fibroblasts were isolated from $\sim 8$-10-week-old mice. Lungs were minced and digested in calcium and magnesium free HBSS buffer supplemented with $10 \mathrm{mg} / \mathrm{ml}$ type I collagenase (Worthington) and $20 \mu \mathrm{g} / \mathrm{ml}$ DNase I (Sigma-Aldrich) for $3 \mathrm{~h}$ at $37{ }^{\circ} \mathrm{C}$ with shaking. Cell suspensions were filtered through sterile mesh and the filtered cells were cultured in DMEM/Ham's F-12 medium containing 10\% FBS, $15 \mathrm{mM}$ HEPES, $20 \mathrm{mM}$ L-glutamine, $50 \mathrm{U} /$ $\mathrm{ml}$ penicillin, and $50 \mu \mathrm{g} / \mathrm{ml}$ streptomycin. Two days later, adherent fibroblasts were rinsed with HBSS and cultured for experiments.

\section{siRNA}

The siRNAs targeting DUBs or ISG15 were synthesized and transfected with Lipofectamine 2000 according to the manufacture's manual. Twenty-four hours after transfection, cells were harvested or stimulated followed by immunoblot, qPCR or deubiquitination assays. The siRNA sequences are listed as follows: Control, 5'-UUCUCCGAACGUGUCACGUTT-3'; siUSP18\#1, 5'-CUGGUUGGUUUACACAACATT-3'; siUSP18 \#2, 5'-GCCCUUGUUUGUCCAACAUTT-3'; siUSP18\#3, 5'-CCGGUCAUUACUGUGUCUATT-3'; siUSP20\#1, 5'-CCAUAGGAGAGGUGACCAATT-3'; siUSP20\#2, 5'-GCCCAUCAGAAGAUGAGUUTT-3'; siUSP20\#3, 5'-GCGUACAAACUCGGAGCAATT-3'; siISG15\#1, 5'-GCACAGUGAUCAAGCAUUUTT-3'; siISG15\#2, 5'-GCAGACUGUAGACACGCUUTT-3'; siISG15\#3, 5'-GGGACCUAAAGGUGAAGAUTT-3'.

\section{Viral infection and plaque assays}

For qRT-PCR or immunoblot analysis, cells seeded into 24well plates $\left(2-5 \times 10^{5}\right.$ cells per well $)$ or six-well plates $\left(10^{6}\right.$ to $10^{7}$ cells per well) were infected with various viruses for the indicated time points. For viral replication assays, cells $\left(2-5 \times 10^{5}\right)$ were infected with HSV-1 or HSV-1-GFP. One hour later, the superna- tants were removed and cells were washed with pre-warmed PBS ( $1 \mathrm{ml})$ twice followed by culture in full medium for $24 \mathrm{~h}$. Viral replication was analyzed by flow cytometry or qRT-PCR analysis. For mouse infection, age- and sex-matched Usp $18^{+/+}$and $U_{s p} 18^{-/-}$ littermates were injected with HSV-1 $\left(8 \times 10^{5}\right.$ PFU per mouse $)$ and the survival of animals was monitored every day. Usp $18^{+/+}$Ifnar $1^{-/}$ and Usp $18^{-/-}$Ifnar $1^{-/-}$were infected with HSV-1 $\left(5 \times 10^{4} \mathrm{PFU}\right.$ per mice). The sera were collected for ELISA analysis at $12 \mathrm{~h}$ and lungs or brains were collected for qRT-PCR or histological analysis at 24 h or 4 days after infection.

For plaque assays, Usp $18^{+/+}$and $U s p 18^{-/-}$littermates were intravenously injected with HSV $-1\left(4 \times 10^{6}\right.$ PFU per mouse $)$ and the kidneys were collected and homogenized in PBS at day 4 after infection. Usp $18^{+/+}$and Usp $18^{-/-}$BMDCs or MEFs $\left(2-5 \times 10^{5}\right)$ were infected with HSV-1 (MOI $=0.05)$. One hour later, the viruses were removed and the cells were washed with pre-warmed PBS twice and cultured in full medium for $36-48 \mathrm{~h}$. The homogenizes of kidneys and supernatants of cell cultures were serially diluted to infect Vero cells. One hour later, the cells were washed with PBS twice and cultured in DMEM containing 8\% FBS, 1\% methylcellulose and $1 \%$ streptomycin and penicillin for $48 \mathrm{~h}$. Cells were fixed with $4 \%$ paraformaldehyde for $15 \mathrm{~min}$ and stained with $0.1 \%$ crystal violet for $20 \mathrm{~min}$.

\section{Lentivirus -mediated gene transfer}

HEK293 cells were transfected with phage-6tag-STING, phage-6tag-USP18, phage-6tag- USP18 (C61A) or the empty vector along with the packaging vectors psPAX2 and pMD2G. The medium was changed with fresh full medium (10\% FBS, $1 \%$ streptomycin-penicillin and $10 \mu \mathrm{M} \beta$-mercaptoethanol) $8 \mathrm{~h}$ after transfection. Forty hours later, the supernatants were harvested to infect MEFs followed by various analyses.

\section{Statistical analysis}

Differences between experimental and control groups were tested using Student's $t$-test or two-way ANOVA with Bonferroni's post-test. $P$ values $<0.05$ were considered statistically significant. For animal survival analysis, the Kaplan-Meier method was adopted to generate graphs, and the survival curves were analyzed with log-rank analysis.

\section{Acknowledgments}

We thank Dr Zhijian Chen for reagents, Drs Yan Zhou, Baoliang Song, members of Zhong lab and the core facilities of College of Life Sciences for technical help. This work was supported by grants from the Ministry of Science and Technology of China (2014CB542601 and 2016YFA0502102), the National Natural Science Foundation of China (31371427, 31521091, 31671454 , and 31622036), the Ministry of Education of China (201427), and State Key Laboratory of Veterinary Etiological Biology (SKLVEB2015KFKT001).

\section{Author Contributions}

BZ designed and supervised the study; MZ, MXZ, QZ, GFZ, LY and BZ performed the experiments; QZ, DE Z, JY and HBS provided reagents; $\mathrm{MZ}, \mathrm{MXZ}$ and $\mathrm{BZ}$ wrote the manuscript; all the authors analyzed data. 
Competing Financial Interests

The authors declare no competing financial interests.

\section{References}

1 Takeuchi O, Akira S. Pattern recognition receptors and inflammation. Cell 2010; 140:805-820.

$2 \mathrm{Wu}$ J, Chen ZJ. Innate immune sensing and signaling of cytosolic nucleic acids. Annu Rev Immunol 2014; 32:461-488.

3 Hou F, Sun L, Zheng H, Skaug B, Jiang QX, Chen ZJ. MAVS forms functional prion-like aggregates to activate and propagate antiviral innate immune response. Cell 2011; 146:448461.

4 Seth RB, Sun L, Ea CK, Chen ZJ. Identification and characterization of MAVS, a mitochondrial antiviral signaling protein that activates NF-kappaB and IRF 3. Cell 2005; 122:669682.

5 Xu LG, Wang YY, Han KJ, Li LY, Zhai Z, Shu HB. VISA is an adapter protein required for virus-triggered IFN-beta signaling. Mol Cell 2005; 19:727-740.

6 Kawai T, Takahashi K, Sato S, et al. IPS-1, an adaptor triggering RIG-I- and Mda5-mediated type I interferon induction. Nat Immunol 2005; 6:981-988.

7 Meylan E, Curran J, Hofmann K, et al. Cardif is an adaptor protein in the RIG-I antiviral pathway and is targeted by hepatitis C virus. Nature 2005; 437:1167-1172.

8 Ablasser A, Bauernfeind F, Hartmann G, Latz E, Fitzgerald KA, Hornung V. RIG-I-dependent sensing of poly(dA:dT) through the induction of an RNA polymerase III-transcribed RNA intermediate. Nat Immunol 2009; 10:1065-1072.

9 Chiu YH, Macmillan JB, Chen ZJ. RNA polymerase III detects cytosolic DNA and induces type I interferons through the RIG-I pathway. Cell 2009; 138:576-591.

10 Takaoka A, Wang Z, Choi MK, et al. DAI (DLM-1/ZBP1) is a cytosolic DNA sensor and an activator of innate immune response. Nature 2007; 448:501-505.

11 Unterholzner L, Keating SE, Baran M, et al. IFI16 is an innate immune sensor for intracellular DNA. Nat Immunol 2010; 11:997-1004.

12 Zhang Z, Yuan B, Bao M, Lu N, Kim T, Liu YJ. The helicase DDX41 senses intracellular DNA mediated by the adaptor STING in dendritic cells. Nat Immunol 2011; 12:959-965.

13 Li Y, Chen R, Zhou Q, et al. LSm14A is a processing body-associated sensor of viral nucleic acids that initiates cellular antiviral response in the early phase of viral infection. Proc Natl Acad Sci USA 2012; 109:11770-11775.

14 Sun L, Wu J, Du F, Chen X, Chen ZJ. Cyclic GMP-AMP synthase is a cytosolic DNA sensor that activates the type I interferon pathway. Science 2013; 339:786-791.

15 Li XD, Wu J, Gao D, Wang H, Sun L, Chen ZJ. Pivotal roles of cGAS-cGAMP signaling in antiviral defense and immune adjuvant effects. Science 2013; 341:1390-1394.

16 Schoggins JW, MacDuff DA, Imanaka N, et al. Pan-viral specificity of IFN-induced genes reveals new roles for cGAS in innate immunity. Nature 2014; 505:691-695.

17 Zhang X, Shi H, Wu J, et al. Cyclic GMP-AMP containing mixed phosphodiester linkages is an endogenous high-affinity ligand for STING. Mol Cell 2013; 51:226-235.

18 Ablasser A, Goldeck M, Cavlar T, et al. cGAS produces a 2'-5'-linked cyclic dinucleotide second messenger that activates STING. Nature 2013; 498:380-384.

19 Zhong B, Yang Y, Li S, et al. The adaptor protein MITA links virus-sensing receptors to IRF3 transcription factor activation. Immunity 2008; 29:538-550.

20 Ishikawa H, Barber GN. STING is an endoplasmic reticulum adaptor that facilitates innate immune signalling. Nature 2008; 455:674-678.

21 Jin L, Hill KK, Filak H, et al. MPYS is required for IFN response factor 3 activation and type I IFN production in the response of cultured phagocytes to bacterial second messengers cyclic-di-AMP and cyclic-di-GMP. J Immunol 2011; 187:2595-2601.

22 Sun W, Li Y, Chen L, et al. ERIS, an endoplasmic reticulum IFN stimulator, activates innate immune signaling through dimerization. Proc Natl Acad Sci USA 2009; 106:8653-8658.

23 Ouyang S, Song X, Wang Y, et al. Structural analysis of the STING adaptor protein reveals a hydrophobic dimer interface and mode of cyclic di-GMP binding. Immunity 2012; 36:1073-1086.

24 Yin Q, Tian Y, Kabaleeswaran V, et al. Cyclic di-GMP sensing via the innate immune signaling protein STING. Mol Cell 2012; 46:735-745.

25 Shu C, Yi G, Watts T, Kao CC, Li P. Structure of STING bound to cyclic di-GMP reveals the mechanism of cyclic dinucleotide recognition by the immune system. Nat Struct Mol Biol 2012; 19:722-724.

26 You F, Sun H, Zhou X, et al. PCBP2 mediates degradation of the adaptor MAVS via the HECT ubiquitin ligase AIP4. Nat Immunol 2009; 10:1300-1308.

27 Zhong B, Zhang Y, Tan B, Liu TT, Wang YY, Shu HB. The E3 ubiquitin ligase RNF5 targets virus-induced signaling adaptor for ubiquitination and degradation. J Immunol 2010; 184:6249-6255.

28 Zhong B, Zhang L, Lei C, et al. The ubiquitin ligase RNF5 regulates antiviral responses by mediating degradation of the adaptor protein MITA. Immunity 2009; 30:397-407.

29 Qin Y, Zhou MT, Hu MM, et al. RNF26 temporally regulates virus-triggered type I interferon induction by two distinct mechanisms. PLoS Pathog 2014; 10:e1004358.

30 Zhang J, Hu MM, Wang YY, Shu HB. TRIM32 protein modulates type I interferon induction and cellular antiviral response by targeting MITA/STING protein for K63-linked ubiquitination. J Biol Chem 2012; 287:28646-28655.

31 Tsuchida T, Zou J, Saitoh T, et al. The ubiquitin ligase TRIM56 regulates innate immune responses to intracellular double-stranded DNA. Immunity 2010; 33:765-776.

32 Wang Q, Liu X, Cui Y, et al. The E3 ubiquitin ligase AMFR and INSIG1 bridge the activation of TBK1 kinase by modifying the adaptor STING. Immunity 2014; 41:919-933.

33 Reyes-Turcu FE, Ventii KH, Wilkinson KD. Regulation and cellular roles of ubiquitin-specific deubiquitinating enzymes. Annu Rev Biochem 2009; 78:363-397.

34 Li Z, Wang D, Messing EM, Wu G. VHL protein-interacting deubiquitinating enzyme 2 deubiquitinates and stabilizes HIF-1alpha. EMBO Rep 2005; 6:373-378.

35 Berthouze M, Venkataramanan V, Li Y, Shenoy SK. The deubiquitinases USP33 and USP20 coordinate beta2 adrenergic receptor recycling and resensitization. EMBO J 2009; 
28:1684-1696.

36 Yasunaga J, Lin FC, Lu X, Jeang KT. Ubiquitin-specific peptidase 20 targets TRAF6 and human T cell leukemia virus type 1 tax to negatively regulate NF-kappaB signaling. $J$ Virol 2011; 85:6212-6219.

37 Yuan J, Luo K, Deng M, et al. HERC2-USP20 axis regulates DNA damage checkpoint through Claspin. Nucleic Acids Res 2014; 42:13110-13121.

38 Zhu M, Zhao H, Liao J, Xu X. HERC2/USP20 coordinates CHK1 activation by modulating CLASPIN stability. Nucleic Acids Res 2014; 42:13074-13081.

39 Schwer H, Liu LQ, Zhou L, et al. Cloning and characterization of a novel human ubiquitin-specific protease, a homologue of murine UBP43 (Usp18). Genomics 2000; 65:44-52.

40 Malakhov MP, Malakhova OA, Kim KI, Ritchie KJ, Zhang DE. UBP43 (USP18) specifically removes ISG15 from conjugated proteins. J Biol Chem 2002; 277:9976-9981.

41 Ritchie KJ, Malakhov MP, Hetherington CJ, et al. Dysregulation of protein modification by ISG15 results in brain cell injury. Genes Dev 2002; 16:2207-2212.

42 Ketscher L, Hannss R, Morales DJ, et al. Selective inactivation of USP18 isopeptidase activity in vivo enhances ISG15 conjugation and viral resistance. Proc Natl Acad Sci USA 2015; 112:1577-1582.

43 Malakhova OA, Kim KI, Luo JK, et al. UBP43 is a novel regulator of interferon signaling independent of its ISG15 isopeptidase activity. EMBO J 2006; 25:2358-2367.

44 Kim KI, Yan M, Malakhova O, et al. Ube1L and protein ISGylation are not essential for alpha/beta interferon signaling. Mol Cell Biol 2006; 26:472-479.

45 Knobeloch KP, Utermohlen O, Kisser A, Prinz M, Horak I. Reexamination of the role of ubiquitin-like modifier ISG15 in the phenotype of UBP43-deficient mice. Mol Cell Biol 2005; 25:11030-11034.

46 Liu X, Li H, Zhong B, et al. USP18 inhibits NF-kappaB and NFAT activation during Th17 differentiation by deubiquitinating the TAK1-TAB1 complex. J Exp Med 2013; 210:15751590.

47 Yang Z, Xian H, Hu J, et al. USP18 negatively regulates NF-kappaB signaling by targeting TAK1 and NEMO for deubiquitination through distinct mechanisms. Sci Rep 2015; 5:12738

48 Ritchie KJ, Hahn CS, Kim KI, et al. Role of ISG15 protease UBP43 (USP18) in innate immunity to viral infection. Nat Med 2004; 10:1374-1378.
49 Honke N, Shaabani N, Cadeddu G, et al. Enforced viral replication activates adaptive immunity and is essential for the control of a cytopathic virus. Nat Immunol 2012; 13:51-57.

50 Donley DK. TORCH infections in the newborn. Semin Neurol 1993; 13:106-115.

51 Meuwissen ME, Schot R, Buta S, et al. Human USP18 deficiency underlies type 1 interferonopathy leading to severe pseudo-TORCH syndrome. J Exp Med 2016; 213:1163-1174.

52 Basters A, Geurink PP, El Oualid F, et al. Molecular characterization of ubiquitin-specific protease 18 reveals substrate specificity for interferon-stimulated gene 15. FEBS J 2014; 281:1918-1928.

53 Malakhova OA, Yan M, Malakhov MP, et al. Protein ISGylation modulates the JAK-STAT signaling pathway. Genes Dev 2003; 17:455-460.

54 Cai X, Chiu YH, Chen ZJ. The cGAS-cGAMP-STING pathway of cytosolic DNA sensing and signaling. Mol Cell 2014; 54:289-296.

$55 \mathrm{Wu}$ J, Sun L, Chen X, et al. Cyclic GMP-AMP is an endogenous second messenger in innate immune signaling by cytosolic DNA. Science 2013; 339:826-830.

56 You F, Wang P, Yang L, et al. ELF4 is critical for induction of type I interferon and the host antiviral response. Nat Immunol 2013; 14:1237-1246.

57 Holm CK, Rahbek SH, Gad HH, et al. Influenza A virus targets a cGAS-independent STING pathway that controls enveloped RNA viruses. Nat Commun 2016; 7:10680.

58 Kim KI, Malakhova OA, Hoebe K, Yan M, Beutler B, Zhang DE. Enhanced antibacterial potential in UBP43-deficient mice against Salmonella typhimurium infection by up-regulating type I IFN signaling. J Immunol. 2005; 175:847-854.

59 Zhou Q, Lin H, Wang S, et al. The ER-associated protein ZDHHC1 is a positive regulator of DNA virus-triggered, MITA/STING-dependent innate immune signaling. Cell Host Microbe 2014; 16:450-461.

60 Lin D, Zhang M, Zhang MX, et al. Induction of USP25 by viral infection promotes innate antiviral responses by mediating the stabilization of TRAF3 and TRAF6. Proc Natl Acad Sci USA 2015; 112:11324-11329.

61 Zhong B, Liu X, Wang X, et al. Ubiquitin-specific protease 25 regulates TLR4-dependent innate immune responses through deubiquitination of the adaptor protein TRAF3. Sci Signal 2013; 6:ra35.

(Supplementary information is linked to the online version of the paper on the Cell Research website.) 